\title{
Global Existence and Optimal Decay Rates for Three-Dimensional Compressible Viscoelastic Flows System with Damping
}

\author{
Wenfeng Xu \\ College of Science, University of Shanghai for Science and Technology, Shanghai, China \\ Email: 18326892899@139.com
}

How to cite this paper: Xu, W.F. (2018) Global Existence and Optimal Decay Rates for Three-Dimensional Compressible Viscoelastic Flows System with Damping. Journal of Applied Mathematics and Physics, 6, 2273-2294.

https://doi.org/10.4236/jamp.2018.611189

Received: October 22, 2018

Accepted: November 16, 2018

Published: November 19, 2018

Copyright $\odot 2018$ by author and Scientific Research Publishing Inc. This work is licensed under the Creative Commons Attribution International License (CC BY 4.0).

http://creativecommons.org/licenses/by/4.0/

\begin{abstract}
In this paper, we consider the large time behavior of the strong solutions to the three dimensional compressible viscoelastic flows with damping. Based on the energy method and spectral analysis, we analyze the influences of the damping on the global existence and decay rates of compressible viscoelastic flows under some small assumptions in $H^{\beta}$-framework. Compared with the time decay rates of solutions to the compressible viscoelastic flows in [1], our results imply that the friction of the damping is stronger than the dissipation effect of the viscosities.
\end{abstract}

\section{Keywords}

Global Existence, Damping, Optimal Decay Rates, Decomposition

\section{Introduction}

In this paper, we are interested in three-dimensional compressible viscoelastic flows with damping in the following form:

$$
\left\{\begin{array}{l}
\rho_{t}+\operatorname{div}(\rho u)=0, \\
(\rho u)_{t}+\operatorname{div}(\rho u \otimes u)+\nabla P(\rho)-\mu \Delta u-(\lambda+\mu) \nabla \operatorname{div} u=\alpha \operatorname{div}\left(\rho F F^{\mathrm{T}}\right)-\rho u,(1.1) \\
F_{t}+u \cdot \nabla F=\nabla u F,
\end{array}\right.
$$

for $(t, x) \in[0,+\infty) \times \mathbb{R}^{3}$. Here $\rho, u \in \mathbb{R}^{3}, F \in M^{3 \times 3}$ denote the density, the velocity and the deformation gradient, respectively. The constants $\mu$ and $\lambda$ are the shear viscosity and the bulk viscosity coefficients of the fluid, respectively, which satisfy

$$
\mu>0, \quad 2 \mu+3 \lambda \geq 0
$$


The corresponding elastic energy is chosen to be the special form of the Hookean linear elastic [2],

$$
W(F)=\frac{\alpha}{2}|F|^{2}+\frac{1}{\rho} \int_{0}^{\rho} P(s) \mathrm{d} s, \quad \alpha>0 .
$$

In this paper, we investigate the Cauchy problem of system (1.1) with the initial data

$$
\left.(\rho, u, F)\right|_{t=0}=\left(\rho_{0}(x), u_{0}(x), F_{0}(x)\right), \quad x \in \mathbb{R}^{3},
$$

with

$$
\operatorname{div}\left(\rho F^{\mathrm{T}}\right)=0, \quad F^{l k}(0) \nabla_{l} F^{i j}(0)=F^{l j}(0) \nabla_{l} F^{i k}(0) .
$$

It is standard that the condition (1.3) is preserved by the flow, which has been proved in [3] [4].

The system (1.1) is a coupling system between Navier-Stokes equations and the deformation gradient with a damping term. When the damping term is absence in the system (1), there are many results about the global existence of solution to the compressible viscoelastic flows, refer to [5] [6] [7]. Hu and Wang in [1] [8] have proved the global existence and the decay rates of multi-dimensional compressible viscoelastic fluids in the large time behavior. The global existence of the Cauchy problem with initial data close to an equilibrium state in Besov spaces was obtained in [3]. For the Navier-Stokes equations, there are many mathematical results on the global existence of the solutions in [9] [10]. Recently, for the Navier-Stokes equations with the electric potential, Wang in [11] proved the global existence of strong solution. They also obtained the decay rates $\left\|\nabla^{k}(\rho-1)\right\|_{L^{2}} \leq C(1+t)^{-\frac{3}{4}-\frac{k+1}{2}}$, which are faster than the decay rates $\left\|\nabla^{k}(\rho-1)\right\|_{L^{2}} \leq C(1+t)^{-\frac{3}{4}-\frac{k}{2}}$ in [1]. For the classical solutions to the incompressible viscoelastic flows, refer to [12]-[18]. And the global existence of weak solutions to the incompressible viscoelastic flows with large initial data is established in [3] [16] [19].

When we consider the damping term, there is very little progress on the global existence of solutions. And for the Navier-Stokes equations with the damping term $\alpha|u|^{\beta-1} u(\alpha>0)$, we refer to [20] [21] [22] [23] [24]. In this paper, we consider the global existence and $L^{2}$-norm decay rates of the compressible viscoelastic flows with the term for $\beta=1$ in $H^{\beta}$ framework. We use the standard energy method to prove the global existence under the condition that the initial data are close to the constant equilibrium state. In order to prove the decay rates, we take the Hodge decomposition of the linear system, and then it becomes two similar systems which only involve two variables. It is different from the compressible viscoelastic fluids [1] [8], we divide the solution $U(n, v, E)(x, t)$ into two parts, the low frequency part $U_{l}$ and the high frequency part $U_{h}$. Here, the key point is the highest order derivative estimates of the high frequency part can be governed by the decay estimates of the low frequency part, see Section 3 . 
We use the energy method for the high frequency part and combine with the decay estimates of $U_{l}$ to obtain the decay estimates of the solutions. Here, when the damping term is taken into account, we obtain the improved global existence of the solutions for system (1.1).

Our main results are stated in the following theorem.

Theorem 1.1 Assume that the initial condition

$\left(\rho_{0}-1, u_{0}, F_{0}-I\right) \in H^{3}\left(\mathbb{R}^{3}\right)$ satisfies the constraints (1.3), there exists a constant $\delta_{0}>0$ such that if

$$
\left\|\left(\rho_{0}-1, u_{0}, F_{0}-I\right)\right\|_{H^{3}\left(\mathbb{R}^{3}\right)} \leq \delta_{0},
$$

then there exists a unique global solution $(\rho, u, F)$ of the Cauchy problem (1.1)-(1.2) such that for any $t \geq 0$,

$$
\begin{aligned}
& \|(\rho-1, u, F-I)(t)\|_{H^{3}\left(\mathbb{R}^{3}\right)}^{2}+\int_{0}^{t}\|\nabla(\rho, F)(\tau)\|_{H^{2}\left(\mathbb{R}^{3}\right)}^{2}+\|u(\tau)\|_{H^{4}\left(\mathbb{R}^{3}\right)}^{2} \mathrm{~d} \tau \\
& \leq C\left\|\left(\rho_{0}-1, u_{0}, F_{0}-I\right)\right\|_{H^{3}\left(\mathbb{R}^{3}\right)}^{2}
\end{aligned}
$$

Moreover, if $\left(\rho_{0}-1, u_{0}\right) \in L^{1}\left(\mathbb{R}^{3}\right)$, the solution $(\rho, u, F)$ enjoys the following decay-in-time estimates.

$$
\begin{gathered}
\left\|\nabla^{k}(\rho-1, u, F-I)(t)\right\|_{L^{2}\left(\mathbb{R}^{3}\right)} \leq C(1+t)^{-\frac{3}{4}-\frac{k}{2}}, \quad 0 \leq k \leq 3, \\
\|(\rho-1, u, F-I)(t)\|_{L^{\infty}\left(\mathbb{R}^{3}\right)} \leq C(1+t)^{-\frac{3}{2}} \\
\left\|\partial_{t}(\rho-1, u, F-I)(t)\right\|_{L^{2}\left(\mathbb{R}^{3}\right)} \leq C(1+t)^{-\frac{3}{4}}
\end{gathered}
$$

for some positive constant $C$.

Notation. We use $L^{p}, W^{m, p}$ to represent the usual Lebesgue and Sobolev spaces on $\mathbb{R}^{3}$ and $H^{m}=W^{m, 2}$, with norms $\|\cdot\|_{L^{p}},\|\cdot\|_{W^{m, p}}$ and $\|\cdot\|_{H^{m}}$, respectively. We also denote $\nabla=\partial_{x}=\left(\partial_{1}, \partial_{2}, \partial_{3}\right)$, where $\partial_{i}=\partial_{x_{i}}, \nabla_{i}=\partial_{i}$. We assume $C$ to be a positive constant throughout this paper that may vary at different places and the integration domain $\mathbb{R}^{3}$ will be always omitted without any ambiguity. Finally $\langle\cdot, \cdot\rangle$ denotes the inner product in $L^{2}\left(\mathbb{R}^{3}\right)$.

The rest of this paper is devoted to prove Theorem 1.1. In Section 2, we reformulate the system and establish a priori estimate for the strong solutions. Then we consider the global existence of strong solutions. In Section 3, we give the decay estimates of the low frequency part and complete the proof of Theorem 1.1. Finally, Section 4 shows some useful inequalities.

\section{Global Existence}

\section{Reformulations}

In this subsection, we first reformulate the system (1.1). For $\rho>0,(1.1)$ can be rewritten as 


$$
\left\{\begin{array}{l}
\rho_{t}+\rho \operatorname{div} u+u \nabla \rho=0, \\
u_{t}^{i}-\frac{1}{\rho}\left(\mu \Delta u^{i}-(\lambda+\mu) \nabla_{i} \operatorname{div} u^{i}\right)+u \cdot \nabla u^{i}+\frac{P^{\prime}(\rho)}{\rho} \nabla_{i} \rho+u^{i}=\alpha F^{j k} \nabla_{j} F^{i k}, \\
F_{t}+u \cdot \nabla F=\nabla u F,
\end{array}\right.
$$

where we use the condition (1.3), $\operatorname{div}\left(\rho F^{\mathrm{T}}\right)=0$, then get the vector $\operatorname{div}\left(\rho F F^{\mathrm{T}}\right)$,

$$
\nabla_{j}\left(\rho F^{i k} F^{j k}\right)=\rho F^{j k} \nabla_{j} F^{i k}+F_{i k} \nabla_{j}\left(\rho F^{j k}\right)=\rho F^{j k} \nabla_{j} F^{i k} .
$$

Let

$$
n(t, x)=\rho-1, v(t, x)=u, E(t, x)=F-I,
$$

then we have from (6) that

$$
\left\{\begin{array}{l}
n_{t}+\operatorname{div} v=f-v \cdot \nabla n, \\
v_{t}^{i}-\mu \Delta v^{i}-(\lambda+\mu) \nabla_{i} \operatorname{div} v^{i}+\nabla_{i} n-\alpha \nabla_{j} E^{i j}+v^{i}=g^{i}, \quad i=1,2,3, \\
E_{t}-\nabla v=h-v \cdot \nabla E,
\end{array}\right.
$$

where

$$
\begin{gathered}
f=-n \nabla \cdot v, \quad h=\nabla v E, \quad \frac{1}{P^{\prime}(1)}=1, \\
g^{i}=\alpha E^{j k} \nabla_{j} E^{i k}-v \cdot \nabla v^{i}-\left(\frac{P^{\prime}(n+1)}{(n+1) P^{\prime}(1)}-1\right) \nabla_{i} n \\
-\frac{n}{n+1}\left(\mu \Delta v^{i}-(\lambda+\mu) \nabla_{i} \operatorname{div} v^{i}\right) .
\end{gathered}
$$

And here $\frac{P^{\prime}(n+1)}{(n+1) P^{\prime}(1)}-1 \sim o(1) n$. We will assume that $\alpha=1$ for the rest of this paper.

Proposition 2.1 (Local existence). Let $\left(n_{0}, v_{0}, E_{0}\right) \in H^{3}\left(\mathbb{R}^{3}\right)$ and $\inf _{\in \in \mathbb{R}^{3}}\left\{\rho_{0}+1\right\}>0$. Then there exists a positive constant $T>0$ depending on $\left(n_{0}, v_{0}, E_{0}\right)$, such that the initial value problem (2.1) has a unique solution $(n, v, E)$ satisfying

$$
\begin{gathered}
n, E \in C^{0}\left(0, T ; H^{3}\left(\mathbb{R}^{3}\right)\right) \cap C^{1}\left(0, T ; H^{2}\left(\mathbb{R}^{3}\right)\right), \\
v \in C^{0}\left(0, T ; H^{4}\left(\mathbb{R}^{3}\right)\right) \cap C^{1}\left(0, T ; L^{2}\left(\mathbb{R}^{3}\right)\right) .
\end{gathered}
$$

Moreover, the following estimates hold,

$$
\sup _{0 \leq t \leq T}\|(n, v, E)(t)\|_{H^{3}}^{2} \leq 3\left\|\left(n_{0}, v_{0}, E_{0}\right)\right\|_{H^{3}}^{2},
$$

and

$$
\inf _{x \in \mathbb{R}^{3}, 0 \leq t \leq T}\{\rho(x, t)+1\}>0,
$$

where $\left(n_{0}, v_{0}, E_{0}\right):=\left(\rho_{0}-1, u_{0}, F_{0}-I\right)$.

Proof. The proof can be done by using the standard iteration arguments. Refer, instance, to [8] [25]. 
Proposition 2.2 (A priori estimate). Assume that

$$
\|(n, v, E)\|_{H^{3}} \leq \delta \ll 1,
$$

then there exists a unique global solution $(n, v, E)$ of the Cauchy problem (1.1)-(1.2) such that for any $t \geq 0$,

$$
\begin{aligned}
& \|(n, v, E)(t)\|_{H^{3}\left(\mathbb{R}^{3}\right)}^{2}+\int_{0}^{t}\|\nabla(v, E)(\tau)\|_{H^{2}\left(\mathbb{R}^{3}\right)}^{2}+\|v(\tau)\|_{H^{4}\left(\mathbb{R}^{3}\right)}^{2} \mathrm{~d} \tau \\
& \leq C\left\|\left(n_{0}, v_{0}, E_{0}\right)\right\|_{H^{3}\left(\mathbb{R}^{3}\right)}^{2} .
\end{aligned}
$$

Moreover, if $\left(n_{0}, v_{0}\right) \in L^{1}\left(\mathbb{R}^{3}\right)$, the solution $(n, v, E)$ enjoys the following decay-in-time estimates.

$$
\begin{gathered}
\left\|\nabla^{k}(n, v, E)(t)\right\|_{L^{2}\left(\mathbb{R}^{3}\right)} \leq C(1+t)^{-\frac{3}{4}-\frac{k}{2}}, \quad 0 \leq k \leq 3, \\
\|(n, v, E)(t)\|_{L^{\infty}\left(\mathbb{R}^{3}\right)} \leq C(1+t)^{-\frac{3}{2}}, \\
\left\|\partial_{t}(n, v, E)(t)\right\|_{L^{2}\left(\mathbb{R}^{3}\right)} \leq C(1+t)^{-\frac{3}{4}} .
\end{gathered}
$$

We can get the global existence of solution by combining the local existence result with a priori estimates. In the following lemma we give the energy estimates of the lower and higher for $(n, v, E)$.

Lemma 2.1 Under the assumption (2.4), it holds that

$$
\frac{\mathrm{d}}{\mathrm{d} t}\|(n, v, E)(t)\|_{L^{2}}^{2}+C\|v(t)\|_{H^{1}}^{2} \leq C \delta\|\nabla(n, E)(t)\|_{L^{2}}^{2} .
$$

Proof. Multiplying $(2.2)_{1},(2.2)_{2},(2.2)_{3}$, by $n, v, E$, respectively, and then integrating them over $\mathbb{R}^{3}$, we obtain

$$
\begin{aligned}
& \frac{1}{2} \frac{\mathrm{d}}{\mathrm{d} t}\|(n, v, E)\|_{L^{2}}^{2}+\|v\|_{L^{2}}^{2}+\mu\|\nabla v\|_{L^{2}}^{2}+(\mu+\lambda)\|\nabla \cdot v\|_{L^{2}}^{2} \\
& =\langle n, f-v \cdot \nabla n\rangle+\langle v, g\rangle+\langle E, h-v \cdot \nabla E\rangle .
\end{aligned}
$$

To estimate the left-hand side of (2.7), we can use the first equation in (2.2). The three terms on the right-hand side of the above equation can be estimated as follows.

First, we can use Hölder's inequality and Sobolev's inequality to get

$$
|\langle n, f-v \cdot \nabla n\rangle|=|\langle n, v \cdot \nabla n\rangle| \leq\|n\|_{L^{2}}\|v\|_{L^{3}}\|n\|_{L^{2}} \leq C \delta\|\nabla n\|_{L^{2}}^{2} .
$$

Similar to the proof of (2.8), we get

$$
\begin{aligned}
|\langle E, h-v \cdot \nabla E\rangle| & \leq C\|E\|_{L^{6}}\|\nabla v\|_{L^{2}}\|\nabla E\|_{L^{3}}+\|E\|_{L^{6}}\|v\|_{L^{3}}\|\nabla E\|_{L^{2}} \\
& \leq C \delta\left(\|\nabla E\|_{L^{2}}^{2}+\|\nabla v\|_{L^{2}}^{2}\right) .
\end{aligned}
$$

For the second term of (2.7), we can get

$$
\left|\left\langle v^{i}, g^{i}\right\rangle\right| \leq C\left(\left|\left\langle E^{j k} \nabla_{j} E^{i k}, v^{i}\right\rangle\right|+\left|\left\langle v \cdot \nabla v^{i}, v^{i}\right\rangle\right|+\left|\left\langle\left(\frac{P^{\prime}(n+1)}{(n+1) P^{\prime}(1)}-1\right) \nabla_{i} n, v^{i}\right\rangle\right|\right) \text {. }
$$


We also use Hölder's inequality and Sobolev's inequality to get

$$
\begin{gathered}
\left|\left\langle E^{j k} \nabla_{j} E^{i k}, v^{i}\right\rangle\right| \leq\|E\|_{L^{3}}\|v\|_{L^{6}}\|\nabla E\|_{L^{2}} \leq C \delta\left(\|\nabla E\|_{L^{2}}^{2}+\|\nabla v\|_{L^{2}}^{2}\right), \\
\left|\left\langle v \cdot \nabla v^{i}, v^{i}\right\rangle\right| \leq\|\nabla v\|_{L^{2}}\|v\|_{L^{3}}\|v\|_{L^{6}} \leq C \delta\|\nabla v\|_{L^{2}}^{2}, \\
\mid\left\langle\left(\frac{P^{\prime}(n+1)}{(n+1) P^{\prime}(1)}-1\right) \nabla_{i} n, v^{i}\right\rangle \leq\|n\|_{L^{3}}\|v\|_{L^{6}}\|\nabla n\|_{L^{2}} \leq C \delta\left(\|\nabla n\|_{L^{2}}^{2}+\|\nabla v\|_{L^{2}}^{2}\right) .
\end{gathered}
$$

Substituting (2.11)-(2.13) into (2.10) gives

$$
|\langle v, g\rangle| \leq C \delta\left(\|\nabla(n, E)\|_{L^{2}}^{2}+\|\nabla v\|_{L^{2}}^{2}\right) .
$$

Combining (2.8), (2.9) and (2.14) with (2.7) since $\delta>0$ is sufficiently small. This completes the proof of Lemma 2.1 .

Lemma 2.2 Under the assumption (2.4), it holds that

$$
\frac{\mathrm{d}}{\mathrm{d} t}\|\nabla(n, v, E)(t)\|_{H^{2}}^{2}+C\|\nabla v(t)\|_{H^{3}}^{2} \leq C \delta\|\nabla(n, E)(t)\|_{H^{2}}^{2} .
$$

Proof. For each multi-index $k$ with $1 \leq k \leq 3$, by applying $\nabla^{k}$ to (2.2) and multiplying them by $\nabla^{k} n, \nabla^{k} v$ and $\nabla^{k} E$ respectively, and integrating over $\mathbb{R}^{3}$, we have

$$
\begin{aligned}
& \frac{1}{2} \frac{\mathrm{d}}{\mathrm{d} t}\left\|\nabla^{k}(n, v, E)\right\|_{L^{2}}^{2}+\left\|\nabla^{k} v\right\|_{L^{2}}^{2}+\mu\left\|\nabla^{k+1} v\right\|_{L^{2}}^{2}+(\mu+\lambda)\left\|\nabla^{k+1} \cdot v\right\|_{L^{2}}^{2} \\
& =\left\langle\nabla^{k} n, \nabla^{k}(f-v \cdot \nabla n)\right\rangle+\left\langle\nabla^{k} v, \nabla^{k} g\right\rangle+\left\langle\nabla^{k} E, \nabla^{k}(h-v \cdot \nabla E)\right\rangle \\
& :=I_{1}+I_{2}+I_{3} .
\end{aligned}
$$

Now we estimate the right-hand side of (2.16). Here we know

$$
\begin{aligned}
& \left\langle\nabla^{k} n, \nabla^{k}(f-v \cdot \nabla n)\right\rangle=\left\langle\nabla^{k} f, \nabla^{k} n\right\rangle-\left\langle\nabla^{k}(v \cdot \nabla n), \nabla^{k} n\right\rangle, \\
& \left\langle\nabla^{k} E, \nabla^{k}(h-v \cdot \nabla E)\right\rangle=\left\langle\nabla^{k} h, \nabla^{k} E\right\rangle-\left\langle\nabla^{k}(v \cdot \nabla E), \nabla^{k} E\right\rangle .
\end{aligned}
$$

By Hölder's inequality and Sobolev's inequality, we have

$$
\begin{aligned}
I_{1} & =\left\langle\nabla^{k} n, \nabla^{k}(f-v \cdot \nabla n)\right\rangle \\
& =\left\langle\nabla^{k} n, \nabla^{k} f\right\rangle-\left\langle\nabla^{k} n, \nabla^{k}(v \cdot \nabla n)\right\rangle \\
& =\left\langle\nabla^{k} n, \nabla^{k}(-n \nabla v)\right\rangle-\left\langle\nabla^{k} n,\left[\nabla^{k}, v\right] \cdot \nabla n\right\rangle-\left\langle\nabla^{k} n, v \nabla^{k+1} n\right\rangle \\
& =-\left\langle\nabla^{k} n,\left[\nabla^{k}, n\right] \nabla v\right\rangle-\left\langle\nabla^{k} n, n \nabla^{k+1} v\right\rangle-\left\langle\nabla^{k} n,\left[\nabla^{k}, v\right] \cdot \nabla n\right\rangle-\left\langle\nabla^{k} n, v \nabla^{k+1} n\right\rangle \\
& :=I_{1}^{1}+I_{1}^{2}+I_{1}^{3}+I_{1}^{4} .
\end{aligned}
$$

Next we estimate the terms $I_{1}^{i}(i=1,2,3,4)$ one by one.

$$
\begin{aligned}
I_{1}^{1} & =\left\langle-\nabla^{k} n,\left[\nabla^{k}, n\right] \nabla v\right\rangle \leq\left\|\nabla^{k} n\right\|_{L^{2}}\left(\left\|\nabla^{k} v\right\|_{L^{2}}\|\nabla n\|_{L^{\infty}}+\left\|\nabla^{k} n\right\|_{L^{2}}\|\nabla v\|_{L^{\infty}}\right) \\
& \leq C \delta\left(\left\|\nabla^{k} n\right\|_{L^{2}}^{2}+\left\|\nabla^{k} v\right\|_{L^{2}}^{2}\right) .
\end{aligned}
$$

Similar to the estimate of $I_{1}^{1}$, we obtain 


$$
\begin{gathered}
I_{1}^{2}=\left\langle-\nabla^{k} n, n \nabla^{k+1} v\right\rangle \leq C\|n\|_{L^{\infty}}\left\|\nabla^{k} n\right\|_{L^{2}}\left\|\nabla^{k+1} v\right\|_{L^{2}} \\
\leq C \delta\left(\left\|\nabla^{k} n\right\|_{L^{2}}^{2}+\left\|\nabla^{k+1} v\right\|_{L^{2}}^{2}\right), \\
I_{1}^{3}=\left\langle-\nabla^{k} n,\left[\nabla^{k}, v\right] \nabla n\right\rangle \leq\left\|\nabla^{k} n\right\|_{L^{2}}\left(\left\|\nabla^{k} n\right\|_{L^{2}}\|\nabla v\|_{L^{\infty}}+\left\|\nabla^{k} v\right\|_{L^{2}}\|\nabla n\|_{L^{\infty}}\right) \\
\leq C \delta\left(\left\|\nabla^{k} n\right\|_{L^{2}}^{2}+\left\|\nabla^{k} v\right\|_{L^{2}}^{2}\right) \\
I_{1}^{4}=\left\langle-\nabla^{k} n, v \nabla^{k+1} n\right\rangle \leq \frac{1}{2}\left|\left\langle\operatorname{div} v,\left|\nabla^{k} n\right|^{2}\right\rangle\right| \leq C\|\nabla v\|_{L^{\infty}}\left\|\nabla^{k} n\right\|_{L^{2}}^{2} \leq C \delta\left\|\nabla^{k} n\right\|_{L^{2}}^{2} .
\end{gathered}
$$

So we can get

$$
I_{1} \leq C \delta\left(\left\|\nabla^{k} n\right\|_{L^{2}}^{2}+\left\|\nabla^{k} v\right\|_{L^{2}}^{2}+\left\|\nabla^{k+1} v\right\|_{L^{2}}^{2}\right) .
$$

Next, we deal with the term $I_{2}$ and $I_{3}$.

$$
\begin{aligned}
\left\langle\nabla^{k} v^{i}, \nabla^{k} g^{i}\right\rangle= & \left\langle\nabla^{k}\left(E^{j k} \nabla_{j} E^{i k}\right), \nabla^{k} v^{i}\right\rangle-\left\langle\nabla^{k}\left(v^{i} \cdot \nabla v^{i}\right), \nabla^{k} v^{i}\right\rangle \\
& -\left\langle\nabla^{k}\left[\left(\frac{P^{\prime}(n+1)}{(n+1) P^{\prime}(1)}-1\right) \nabla_{i} n\right], \nabla^{k} v^{i}\right\rangle \\
& +\left\langle\nabla^{k}\left(\frac{n}{n+1} \mu \Delta v^{i}+\frac{n}{n+1}(\mu+\lambda) \nabla_{i} \operatorname{div} v\right), \nabla^{k} v\right\rangle \\
& :=I_{2}^{1}+I_{2}^{2}+I_{2}^{3}+I_{2}^{4}+I_{2}^{5} .
\end{aligned}
$$

And we have

$$
\begin{aligned}
I_{2}^{1} & =\left\langle\nabla^{k}\left(E^{j k} \nabla_{j} E^{i k}\right), \nabla^{k} v^{i}\right\rangle \\
& =\left\langle\nabla^{k} E^{j k} \nabla_{j} E^{i k}, \nabla^{k} v^{i}\right\rangle+\left\langle E^{j k}\left[\nabla^{k}, \nabla_{j} E^{i k}\right], \nabla^{k} v^{i}\right\rangle \\
& :=I_{2}^{1,1}+I_{2}^{1,2} .
\end{aligned}
$$

Similarly, by using $(2.2)_{3}$, we have

$$
\begin{aligned}
I_{2}^{1,1} & =\left\langle\nabla^{k} E^{j k} \nabla_{j} E^{i k}, \nabla^{k} v^{i}\right\rangle \leq C\left\|\nabla^{k} E^{j k}\right\|_{L^{2}}\left\|\nabla_{j} E^{i k}\right\|_{L^{2}}\left\|\nabla^{k} v^{i}\right\|_{L^{6}} \\
& \leq C \delta\left(\left\|\nabla^{k} E^{j k}\right\|_{L^{2}}^{2}+\left\|\nabla^{k+1} v^{i}\right\|_{L^{2}}^{2}\right) .
\end{aligned}
$$

Similar to the proof of $I_{1}^{2,1}$, we have

$$
\begin{aligned}
I_{2}^{1,2}= & \left\langle E^{j k}\left[\nabla^{k}, \nabla_{j} E^{i k}\right], \nabla^{k} v^{i}\right\rangle=-\left\langle\left[\nabla^{k}, E^{i k}\right], \nabla\left(E^{j k} \nabla^{k} v^{i}\right)\right\rangle \\
= & -\left\langle\left[\nabla^{k}, E^{i k}\right], \nabla E^{j k} \nabla^{k} v^{i}\right\rangle-\left\langle\left[\nabla^{k}, E^{i k}\right], E^{j k} \nabla^{k+1} v^{i}\right\rangle \\
\leq & \left.C\left\|\nabla^{k} v^{i}\right\|_{L^{2}}\left\|\nabla^{k} E^{j k}\right\|_{L^{2}}\left\|\nabla E^{i k}\right\|_{L^{\infty}}\right) \\
& +C \delta\left\|\nabla^{k+1} v^{i}\right\|_{L^{2}}\left(\left\|\nabla^{k-1} E^{j k}\right\|_{L^{6}}\left\|\nabla E^{i k}\right\|_{L^{3}}+\left\|\nabla^{k} E^{i k}\right\|_{L^{2}}\left\|E^{j k}\right\|_{L^{\infty}}\right) \\
\leq & C \delta\left(\left\|\nabla^{k} v^{i}\right\|_{L^{2}}^{2}+\left\|\nabla^{k} E^{j k}\right\|_{L^{2}}^{2}+\left\|\nabla^{k+1} v^{i}\right\|_{L^{2}}^{2}\right) .
\end{aligned}
$$

So we get

$$
I_{2}^{1} \leq C \delta\left(\left\|\nabla^{k} v^{i}\right\|_{L^{2}}^{2}+\left\|\nabla^{k} E^{j k}\right\|_{L^{2}}^{2}+\left\|\nabla^{k+1} v^{i}\right\|_{L^{2}}^{2}\right) .
$$

Similarly, we can estimate the other terms. 


$$
\begin{aligned}
I_{2}^{2} & =-\left\langle\nabla^{k}\left(v \cdot \nabla v^{i}\right), \nabla^{k} v^{i}\right\rangle \\
& =-\left\langle\left[\nabla^{k}, v\right] \nabla v^{i}, \nabla^{k} v^{i}\right\rangle-\left\langle v \nabla^{k} \nabla v^{i}, \nabla^{k} v^{i}\right\rangle \\
& =-\left\langle\left[\nabla^{k}, v\right] \nabla v^{i}, \nabla^{k} v^{i}\right\rangle+\frac{1}{2}\left\langle\operatorname{div} v,\left|\nabla^{k} v^{i}\right|^{2}\right\rangle \\
& \leq C\left\|\nabla^{k} v^{i}\right\|_{L^{2}}\left(\left\|\nabla^{k} v^{i}\right\|_{L^{2}}\left\|\nabla v^{i}\right\|_{L^{\infty}}\right)+C\|\operatorname{div} v\|_{L^{\infty}}\left\|\nabla^{k} v^{i}\right\|_{L^{2}}^{2} \\
& \leq C \delta\left\|\nabla^{k} v\right\|_{L^{2}}^{2} .
\end{aligned}
$$

For term $I_{2}^{3}$, we have

$$
\begin{aligned}
I_{2}^{3} & =-\left\langle\nabla^{k}\left[\left(\frac{P^{\prime}(n+1)}{(n+1) P^{\prime}(1)}-1\right) \nabla_{i} n\right], \nabla^{k} v^{i}\right\rangle \\
& =-\left\langle\nabla^{k}\left[n \nabla_{i} n\right], \nabla^{k} v^{i}\right\rangle \\
& =-\left\langle\left[\nabla^{k}, n\right] \nabla_{i} n, \nabla^{k} v^{i}\right\rangle-\left\langle n \nabla^{k} \nabla_{i} n, \nabla^{k} v^{i}\right\rangle \\
& :=I_{2}^{3,1}+I_{2}^{3,2} .
\end{aligned}
$$

Then we have

$$
\begin{aligned}
I_{2}^{3,1} & =-\left\langle\left[\nabla^{k}, n\right] \nabla_{i} n, \nabla^{k} v^{i}\right\rangle \leq C\left\|\nabla^{k} v\right\|_{L^{2}}\left(\left\|\nabla^{k} n\right\|_{L^{2}}\|\nabla n\|_{L^{\infty}}\right) \\
& \leq C \delta\left(\left\|\nabla^{k} v\right\|_{L^{2}}^{2}+\left\|\nabla^{k} n\right\|_{L^{2}}^{2}\right) .
\end{aligned}
$$

And same as $I_{1}^{2}$, we have

$$
\begin{aligned}
I_{2}^{3,2} & =-\left\langle n \nabla^{k} \nabla_{i} n, \nabla^{k} v^{i}\right\rangle=\left\langle\nabla^{k} n, \nabla\left(n \nabla^{k} v^{i}\right)\right\rangle \\
& =\left\langle\nabla^{k} n, \nabla n \nabla^{k} v^{i}\right\rangle+\left\langle\nabla^{k} n, n \nabla^{k+1} v^{i}\right\rangle \\
& \leq C \delta\left(\left\|\nabla^{k} n\right\|_{L^{2}}^{2}+\left\|\nabla^{k} v^{i}\right\|_{L^{2}}^{2}+\left\|\nabla^{k+1} v^{i}\right\|_{L^{2}}^{2} .\right.
\end{aligned}
$$

Thus we can give the estimates of the $I_{2}$,

$$
I_{2} \leq C \delta\left(\left\|\nabla^{k} n\right\|_{L^{2}}^{2}+\left\|\nabla^{k} v\right\|_{L^{2}}^{2}+\left\|\nabla^{k} E\right\|_{L^{2}}^{2}+\left\|\nabla^{k+1} v\right\|_{L^{2}}^{2}\right) .
$$

Finally we can estimate the term of $I_{3}$ same as $I_{1}$,

$$
I_{3} \leq C \delta\left(\left\|\nabla^{k} v\right\|_{L^{2}}^{2}+\left\|\nabla^{k} E\right\|_{L^{2}}^{2}+\left\|\nabla^{k+1} v\right\|_{L^{2}}^{2}\right) .
$$

Substituting (2.22), (2.30) and (2.31) into (2.16), we get (2.15).

In the following lemmas we give the dissipations on $\|\nabla n\|_{H^{2}}$ and $\left\|\nabla\left(E^{\mathrm{T}}-E\right)\right\|_{H^{2}}$.

Lemma 2.3 Under the assumption (2.4), there exists a positive constant $C$ such that

$$
\begin{gathered}
-\frac{\mathrm{d}}{\mathrm{d} t}\langle\operatorname{div} v, n\rangle+C\|\nabla n\|_{L^{2}}^{2} \leq C\|v\|_{H^{2}}^{2}+C \delta\|\nabla E\|_{L^{2}}^{2}, \\
\frac{\mathrm{d}}{\mathrm{d} t}\langle\operatorname{div} v, \Delta n\rangle+C\left\|\nabla^{2} n\right\|_{L^{2}}^{2} \leq C\|v\|_{H^{3}}^{2}+C \delta\left\|\nabla^{2} E\right\|_{L^{2}}^{2}, \\
\frac{\mathrm{d}}{\mathrm{d} t}\left\langle\nabla^{2} v, \nabla^{3} n\right\rangle+C\left\|\nabla^{3} n\right\|_{L^{2}}^{2} \leq C\|v\|_{H^{4}}^{2}+C \delta\left\|\nabla^{3} E\right\|_{L^{2}}^{2} .
\end{gathered}
$$

Proof. Notice that the condition $\operatorname{div}\left(\rho F^{\mathrm{T}}\right)=0$ for all $t \geq 0$ gives

$$
\operatorname{divdiv}[(1+n)(E+I)]=0, \quad \forall t \geq 0 .
$$


Thus we get

$$
\begin{aligned}
\frac{\partial^{2}\left(E^{i j}\right)}{\partial_{x i} \partial_{x j}} & =\operatorname{divdiv}\left(E^{\mathrm{T}}\right)=\operatorname{div} \operatorname{div}\left[(1+n)(E+I)^{\mathrm{T}}\right]-\operatorname{div} \operatorname{div}\left(n I+n E^{\mathrm{T}}\right) \\
& =-\Delta n-\operatorname{div} \operatorname{div}(n E) .
\end{aligned}
$$

Then applying $\nabla_{i}$ to $(2.2)_{2}$ and summing over $i$, we can get

$$
(\operatorname{div} v)_{t}-(2 \mu+\lambda) \Delta \operatorname{div} v+2 \Delta n+\nabla v=\operatorname{divg} g_{1}
$$

where

$$
g_{1}=g-\operatorname{div}(n E) .
$$

Multiplying (2.36) by $n$, and then integrating over $\mathbb{R}^{3}$, we get

$$
\begin{aligned}
2\|\nabla n\|_{L^{2}}^{2}= & \left\langle(\operatorname{div} v)_{t}, n\right\rangle+\langle\nabla v, n\rangle-\left\langle\operatorname{divg}_{1}, n\right\rangle-\langle(2 \mu+\lambda) \Delta \operatorname{div} v, n\rangle \\
= & \frac{\mathrm{d}}{\mathrm{d} t}\langle\operatorname{div} v, n\rangle-\left\langle\operatorname{div} v, n_{t}\right\rangle+\langle\nabla v, n\rangle-\left\langle\operatorname{divg}_{1}, n\right\rangle+\langle(2 \mu+\lambda) \Delta \operatorname{div} v, n\rangle \\
= & \frac{\mathrm{d}}{\mathrm{d} t}\langle\operatorname{div} v, n\rangle-\langle\operatorname{div} v, f\rangle+\langle\operatorname{div} v, v \cdot \nabla n\rangle+\|\operatorname{div} v\|_{L^{2}}^{2} \\
& +\langle\nabla v, n\rangle+\left\langle g_{1}, \nabla n\right\rangle+\langle(2 \mu+\lambda) \Delta \operatorname{div} v, n\rangle,
\end{aligned}
$$

where we use the Hölder's, Sobolev's and Cauchy's inequalities to get

$$
\begin{aligned}
& -\frac{\mathrm{d}}{\mathrm{d} t}\langle\operatorname{div} v, n\rangle+2\|\nabla n\|_{L^{2}}^{2} \\
& \leq C\left(\|\nabla v\|_{L^{2}}\|f\|_{L^{2}}+\|\nabla v\|_{L^{2}}\|v\|_{L^{6}}\|\nabla n\|_{L^{3}}+\left\|\nabla^{2} v\right\|_{L^{2}}\|\nabla n\|_{L^{2}}\right. \\
& \left.\quad+\|d i v v\|_{L^{2}}^{2}+\|v\|_{L^{2}}^{2}+\|\nabla n\|_{L^{2}}^{2}+\left\|g_{1}\right\|_{L^{2}}\|\nabla n\|_{L^{2}}\right) \\
& \leq C\|v\|_{H^{2}}^{2}+\frac{1}{2}\|\nabla n\|_{L^{2}}^{2}+C \delta\left(\|\nabla(n, E)\|_{L^{2}}^{2}\right) .
\end{aligned}
$$

Multiplying (2.36) by $\Delta n$, and then integrating over $\mathbb{R}^{3}$, we get

$$
\begin{aligned}
2\|\Delta n\|_{L^{2}}^{2}= & -\left\langle(\operatorname{div} v)_{t}, \Delta n\right\rangle-\langle\nabla v, \Delta n\rangle+\left\langle\operatorname{divg} g_{1}, \Delta n\right\rangle+\langle(2 \mu+\lambda) \Delta \operatorname{div} v, \Delta n\rangle \\
= & -\frac{\mathrm{d}}{\mathrm{d} t}\langle\operatorname{div} v, \Delta n\rangle+\left\langle\operatorname{div} v, \Delta n_{t}\right\rangle-\langle\nabla v, \Delta n\rangle \\
& +\left\langle\operatorname{divg} g_{1}, \Delta n\right\rangle+\langle(2 \mu+\lambda) \Delta \operatorname{div} v, \Delta n\rangle \\
= & -\frac{\mathrm{d}}{\mathrm{d} t}\langle\operatorname{div} v, \Delta n\rangle+\langle\nabla \operatorname{div} v, \nabla f\rangle-\langle\operatorname{div} v, \Delta(v \cdot \nabla n)\rangle+\|\nabla \operatorname{div} v\|_{L^{2}}^{2} \\
& -\langle\nabla v, \Delta n\rangle+\left\langle\operatorname{divg}_{1}, \Delta n\right\rangle+\langle(2 \mu+\lambda) \Delta \operatorname{div} v, \Delta n\rangle .
\end{aligned}
$$

By Hölder's, Sobolev's and Cauchy's inequalities we get

$$
\begin{aligned}
& \frac{\mathrm{d}}{\mathrm{d} t}\langle\operatorname{div} v, \Delta n\rangle+\left\|\nabla^{2} n\right\|_{L^{2}}^{2} \\
& \leq C\left(\left\|\nabla^{2} v\right\|_{L^{2}}\|\nabla f\|_{L^{2}}+\left\|\nabla^{3} v\right\|_{L^{2}}\|v\|_{L^{6}}\|\nabla n\|_{L^{3}}+\left\|\nabla^{2} v\right\|_{L^{2}}^{2}\right. \\
& \left.\quad+\|v\|_{L^{2}}^{2}+\left\|\nabla^{2} n\right\|_{L^{2}}^{2}+\left\|\nabla^{3} v\right\|_{L^{2}}\|\Delta n\|_{L^{2}}+\left\|\nabla g_{1}\right\|_{L^{2}}\|\Delta n\|_{L^{2}}\right) \\
& \leq C\|v\|_{H^{3}}^{2}+\frac{1}{2}\|\Delta n\|_{L^{2}}^{2}+C \delta\left(\left\|\nabla^{2}(n, E)\right\|_{L^{2}}^{2}\right) .
\end{aligned}
$$


Then applying $\nabla_{i}^{2}$ to $(2.2)_{2}$, summing over $i$ and multiplying them by $\nabla^{3} n$, and then integrating them, we have

$$
\frac{\mathrm{d}}{\mathrm{d} t}\left\langle\nabla^{2} v, \nabla^{3} n\right\rangle+\left\|\nabla^{3} n\right\|_{L^{2}}^{2} \leq C\|v\|_{H^{4}}^{2}+C \delta\left(\left\|\nabla^{3}(n, E)\right\|_{L^{2}}^{2}\right),
$$

which gives (2.34) if $\delta$ is small enough. This completes the proof of Lemma 2.3.

Lemma 2.4 Under the assumption (2.4), there exists a positive constant $C$ such that

$$
\begin{gathered}
-\frac{\mathrm{d}}{\mathrm{d} t}\left\langle\mathcal{W}, E^{\mathrm{T}}-E\right\rangle+C\left\|\nabla\left(E^{\mathrm{T}}-E\right)\right\|_{L^{2}}^{2} \leq C\|v\|_{H^{1}}^{2}+C \delta\|\nabla(n, E)\|_{L^{2}}^{2}, \\
\frac{\mathrm{d}}{\mathrm{d} t}\left\langle\mathcal{W}, \Delta\left(E^{\mathrm{T}}-E\right)\right\rangle+C\left\|\nabla^{2}\left(E^{\mathrm{T}}-E\right)\right\|_{L^{2}}^{2} \leq C\|\nabla v\|_{H^{2}}^{2}+C \delta\left\|\nabla^{2}(n, E)\right\|_{L^{2}}^{2}, \\
\frac{\mathrm{d}}{\mathrm{d} t}\left\langle\nabla \mathcal{W}, \nabla^{3}\left(E^{\mathrm{T}}-E\right)\right\rangle+C\left\|\nabla^{3}\left(E^{\mathrm{T}}-E\right)\right\|_{L^{2}}^{2} \leq C\|\nabla v\|_{H^{3}}^{2}+C \delta\left\|\nabla^{3}(n, E)\right\|_{L^{2}}^{2} .
\end{gathered}
$$

Proof. Taking $(2.2)_{3}^{\mathrm{T}}-(2.2)_{3}$, then we have

$$
\left(E^{\mathrm{T}}-E\right)_{t}+\mathcal{W}=h^{\mathrm{T}}-h-v \cdot \nabla\left(E^{\mathrm{T}}-E\right),
$$

where $\mathcal{W}=\nabla v-(\nabla v)^{\mathrm{T}}=\operatorname{curl} v$. Note the condition $F^{l k} \nabla_{l} F^{i j}=F^{l j} \nabla_{l} F^{i k}$ for all $t \geq 0$, which means that

$$
\nabla_{k} E^{i j}+E^{l k} \nabla_{l} E^{i j}=\nabla_{j} E^{i k}+E^{l j} \nabla_{l} E^{i k}, \quad \forall t \geq 0 .
$$

Then we get

$$
\begin{aligned}
& \nabla_{j} \nabla_{k} E^{i k}-\nabla_{i} \nabla_{k} E^{j k}=\nabla_{k} \nabla_{j} E^{i k}-\nabla_{k} \nabla_{i} E^{j k} \\
& =\nabla_{k} \nabla_{k} E^{i j}-\nabla_{k} \nabla_{k} E^{j i}+\nabla_{k}\left(E^{l k} \nabla_{k} E^{i j}-E^{l j} \nabla_{l} E^{i k}\right)-\nabla_{k}\left(E^{l k} \nabla_{l} E^{j i}-E^{l i} \nabla_{l} E^{j k}\right) \\
& =\Delta\left(E^{i j}-E^{j i}\right)+\nabla_{k}\left(E^{l k} \nabla_{l} E^{i j}-E^{l j} \nabla_{l} E^{j k}\right)-\nabla_{k}\left(E^{l k} \nabla_{l} E^{j i}-E^{l i} \nabla_{l} E^{j k}\right) .
\end{aligned}
$$

Apply curlv to $(2.2)_{2}$, we get

$$
\mathcal{W}_{t}-\mu \Delta \mathcal{W}+\mathcal{W}+\Delta\left(E^{\mathrm{T}}-E\right)=\operatorname{curl} g+\mathcal{S},
$$

where the antisymmetric matrix $\mathcal{S}$ is defined as

$$
\mathcal{S}^{i j}=\nabla_{k}\left(E^{l k} \nabla_{l} E^{i j}-E^{l j} \nabla_{l} E^{j k}\right)-\nabla_{k}\left(E^{l k} \nabla_{l} E^{j i}-E^{l i} \nabla_{l} E^{j k}\right) .
$$

Multiplying (2.43) by $\left(E^{\mathrm{T}}-E\right)$, and then integrating over $\mathbb{R}^{3}$, we get

$$
\begin{aligned}
& \left\langle\mathcal{W}, E^{\mathrm{T}}-E\right\rangle+\left\langle\mathcal{W}_{t}, E^{\mathrm{T}}-E\right\rangle+\left\langle\Delta\left(E^{\mathrm{T}}-E\right), E^{\mathrm{T}}-E\right\rangle-\mu\left\langle\Delta \mathcal{W}, E^{\mathrm{T}}-E\right\rangle \\
& =\left\langle\operatorname{curl} g+\mathcal{S}, E^{\mathrm{T}}-E\right\rangle .
\end{aligned}
$$

By Hölder's and Sobolev's inequalities we have

$$
\begin{aligned}
& -\frac{\mathrm{d}}{\mathrm{d} t}\left\langle\mathcal{W}, E^{\mathrm{T}}-E\right\rangle+\left\|\nabla\left(E^{T}-E\right)\right\|_{L^{2}}^{2} \\
& =\left\langle\mathcal{W}, E^{\mathrm{T}}-E\right\rangle-\left\langle\mathcal{W},\left(E^{\mathrm{T}}-E\right)_{t}\right\rangle-\mu\left\langle\Delta \mathcal{W}, E^{\mathrm{T}}-E\right\rangle-\left\langle\operatorname{curl} g+\mathcal{S}, E^{\mathrm{T}}-E\right\rangle,
\end{aligned}
$$

where $\left\langle\mathcal{W},\left(E^{\mathrm{T}}-E\right)_{t}\right\rangle=\langle\mathcal{W},-\mathcal{W}\rangle+\left\langle\mathcal{W}, h^{\mathrm{T}}-h-v \cdot \nabla\left(E^{\mathrm{T}}-E\right)\right\rangle$. Now let us estimate the right-hand side term by term. We use Hölder's and Sobolev's inequa- 
lities have

$$
\begin{gathered}
\left\langle\mathcal{W},\left(E^{\mathrm{T}}-E\right)\right\rangle \mid \leq C\left(\|v\|_{L^{2}}^{2}+\|\nabla E\|_{L^{2}}^{2}\right), \\
|\langle\mathcal{W}, \mathcal{W}\rangle| \leq C\|\nabla v\|_{L^{2}}^{2}, \\
\left|\left\langle\mathcal{W}, h^{T}-h\right\rangle\right| \leq C\|\nabla v\|_{L^{2}}\|\nabla v\|_{L^{2}}\|E\|_{L^{\infty}} \leq C \delta\|\nabla v\|_{L^{2}}^{2}, \\
\left\langle\left\langle\mathcal{W}, v \cdot \nabla\left(E^{\mathrm{T}}-E\right)\right\rangle\right| \leq C\|v\|_{L^{\infty}}\|\nabla v\|_{L^{2}}\|\nabla E\|_{L^{2}} \leq C \delta\|\nabla v\|_{L^{2}}^{2}+C \delta\|\nabla E\|_{L^{2}}^{2}, \\
\left|\mu\left\langle\Delta \mathcal{W}, E^{\mathrm{T}}-E\right\rangle\right|=\mu\left\langle\nabla \mathcal{W}, \nabla\left(E^{\mathrm{T}}-E\right)\right\rangle \leq C\left\|\nabla^{2} v\right\|_{L^{2}}^{2}+C\|\nabla E\|_{L^{2}}^{2}, \\
\left|\left\langle\operatorname{curl} g+\mathcal{S}, E^{\mathrm{T}}-E\right\rangle\right| \leq C \delta\left(\|\nabla v\|_{L^{2}}^{2}+\|\nabla(n, E)\|_{L^{2}}^{2}\right) .
\end{gathered}
$$

Thus we get

$$
-\frac{\mathrm{d}}{\mathrm{d} t}\left\langle\mathcal{W}, E^{\mathrm{T}}-E\right\rangle+C\left\|\nabla\left(E^{\mathrm{T}}-E\right)\right\|_{L^{2}}^{2} \leq C\|v\|_{H^{2}}^{2}+C \delta\|\nabla(n, E)\|_{L^{2}}^{2} .
$$

Similarly, multiplying (2.43) by $\Delta\left(E^{\mathrm{T}}-E\right)$, and then integrating over $\mathbb{R}^{3}$, we get

$$
\begin{aligned}
& \left\langle\mathcal{W}, \Delta\left(E^{\mathrm{T}}-E\right)\right\rangle+\left\langle\mathcal{W}_{t}, \Delta\left(E^{\mathrm{T}}-E\right)\right\rangle+\left\langle\Delta\left(E^{\mathrm{T}}-E\right), \Delta\left(E^{\mathrm{T}}-E\right)\right\rangle \\
& -\mu\left\langle\Delta \mathcal{W}, \Delta\left(E^{\mathrm{T}}-E\right)\right\rangle=\left\langle\operatorname{curlg}+\mathcal{S}, \Delta\left(E^{\mathrm{T}}-E\right)\right\rangle,
\end{aligned}
$$

then

$$
\begin{aligned}
& \left\langle\mathcal{W}, \Delta\left(E^{\mathrm{T}}-E\right)\right\rangle+\frac{\mathrm{d}}{\mathrm{d} t}\left\langle\mathcal{W}, \Delta\left(E^{\mathrm{T}}-E\right)\right\rangle-\left\langle\mathcal{W}, \Delta\left(E^{\mathrm{T}}-E\right)_{t}\right\rangle \\
& -\mu\left\langle\Delta \mathcal{W}, \Delta\left(E^{\mathrm{T}}-E\right)\right\rangle+\left\langle\Delta\left(E^{\mathrm{T}}-E\right), \Delta\left(E^{\mathrm{T}}-E\right)\right\rangle \\
& =\left\langle\operatorname{curl} g+\mathcal{S}, \Delta\left(E^{\mathrm{T}}-E\right)\right\rangle .
\end{aligned}
$$

By Hölder's and Sobolev's inequalities we get

$$
\begin{aligned}
& \frac{\mathrm{d}}{\mathrm{d} t}\left\langle\mathcal{W}, \Delta\left(E^{\mathrm{T}}-E\right)\right\rangle+\left\|\nabla^{2}\left(E^{\mathrm{T}}-E\right)\right\|_{L^{2}}^{2} \\
= & -\left\langle\mathcal{W}, \Delta\left(E^{\mathrm{T}}-E\right)\right\rangle+\left\langle\mathcal{W}, \Delta\left(E^{\mathrm{T}}-E\right)_{t}\right\rangle+\mu\left\langle\Delta \mathcal{W}, \Delta\left(E^{\mathrm{T}}-E\right)\right\rangle \\
& +\left\langle\operatorname{curl}+\mathcal{S}, \Delta\left(E^{\mathrm{T}}-E\right)\right\rangle .
\end{aligned}
$$

Now let us estimate the right-hand side term by term. We use Hölder's and Sobolev's inequalities have

$$
\begin{aligned}
&\left\langle\left\langle\mathcal{W}, \Delta\left(E^{\mathrm{T}}-E\right)\right\rangle\right|=\left|\left\langle\nabla \mathcal{W}, \nabla\left(E^{\mathrm{T}}-E\right)\right\rangle\right| \leq C\left(\left\|\nabla^{2} v\right\|_{L^{2}}+\left\|\nabla^{2} E\right\|_{L^{2}}\right), \\
&|\langle\mathcal{W}, \Delta \mathcal{W}\rangle|=|\langle\nabla \mathcal{W}, \nabla \mathcal{W}\rangle| \leq C\left\|\nabla^{2} v\right\|_{L^{2}}^{2}, \\
&\left\langle\mathcal{W}, \Delta\left(h^{\mathrm{T}}-h\right)\right\rangle \mid=\left|\left\langle\nabla \mathcal{W}, \nabla\left(h^{\mathrm{T}}-h\right)\right\rangle\right| \\
& \leq C\left\|\nabla^{2} v\right\|_{L^{2}}\|\nabla v\|_{L^{2}}\|\nabla E\|_{L^{6}}+C\left\|\nabla^{2} v\right\|_{L^{2}}^{2}\|E\|_{L^{\infty}} \\
& \leq C \delta\left\|\nabla^{2} v\right\|_{L^{2}}^{2}+C \delta\left\|\nabla^{2} E\right\|_{L^{2}}^{2}
\end{aligned}
$$




$$
\begin{aligned}
&\left\langle\left\langle\mathcal{W}, \Delta\left(v \cdot \nabla\left(E^{\mathrm{T}}-E\right)\right)\right\rangle\right|=\left|\left\langle\nabla \mathcal{W}, \nabla\left(v \cdot \nabla\left(E^{\mathrm{T}}-E\right)\right)\right\rangle\right| \\
& \leq C\|\nabla v\|_{L^{2}}\left\|\nabla^{2} v\right\|_{L^{2}}\left\|\nabla\left(E^{\mathrm{T}}-E\right)\right\|_{L^{6}} \\
&+C\|v\|_{L^{\infty}}\left\|\nabla^{2} v\right\|_{L^{2}}\left\|\nabla^{2}\left(E^{\mathrm{T}}-E\right)\right\|_{L^{2}} \\
& \leq C \delta\left\|\nabla^{2} v\right\|_{L^{2}}^{2}+C \delta\left\|\nabla^{2}\left(E^{\mathrm{T}}-E\right)\right\|_{L^{2}}^{2}, \\
&\left|\left\langle\operatorname{curl} g+\mathcal{S}, \Delta\left(E^{\mathrm{T}}-E\right)\right\rangle\right| \leq C \delta\left\|\nabla^{2}\left(E^{\mathrm{T}}-E\right)\right\|_{L^{2}}^{2}+C\left\|\nabla^{2} v\right\|_{H^{1}}^{2}, \\
&\left|\mu\left\langle\Delta \mathcal{W}, \Delta\left(E^{\mathrm{T}}-E\right)\right\rangle\right| \leq C\left\|\nabla^{3} v\right\|_{L^{2}}^{2}+C\left\|\nabla^{2} E\right\|_{L^{2}}^{2} .
\end{aligned}
$$

Similarly, applying $\nabla$ to $(2.43)$ and multiplying by $\nabla^{3}\left(E^{\mathrm{T}}-E\right)$, and then integrating over $\mathbb{R}^{3}$, we get

$$
\frac{\mathrm{d}}{\mathrm{d} t}\left\langle\nabla \mathcal{W}, \nabla^{3}\left(E^{\mathrm{T}}-E\right)\right\rangle+C\left\|\nabla^{3}\left(E^{\mathrm{T}}-E\right)\right\|_{L^{2}}^{2} \leq C\|\nabla v\|_{H^{3}}^{2}+C \delta\left\|\nabla^{3}(n, E)\right\|_{L^{2}}^{2} .
$$

The proof of Lemma 2.4 is completed.

Lemma 2.5 Under the assumption (2.4), it holds that

$$
\begin{gathered}
\|\nabla E\|_{L^{2}}^{2} \leq C\left\|\nabla\left(n, E^{\mathrm{T}}-E\right)\right\|_{L^{2}}^{2}, \\
\left\|\nabla^{2} E\right\|_{L^{2}}^{2} \leq C\left\|\nabla^{2}\left(n, E^{\mathrm{T}}-E\right)\right\|_{L^{2}}^{2}, \\
\left\|\nabla^{3} E\right\|_{L^{2}}^{2} \leq C\left\|\nabla^{3}\left(n, E^{\mathrm{T}}-E\right)\right\|_{L^{2}}^{2} .
\end{gathered}
$$

Proof. Combining (2.35) and (2.42), we get

$$
\begin{aligned}
\Delta \operatorname{div} E & =\nabla \operatorname{div} \operatorname{div} E+\operatorname{divcurldiv} E \\
& =-\Delta \nabla n-\nabla \operatorname{div} \operatorname{div}(n E)-\Delta \operatorname{div}\left(E^{\mathrm{T}}-E\right)-\operatorname{div} \mathcal{S} .
\end{aligned}
$$

Thus using the property of the Riesz potential, from (2.4), we have

$$
\operatorname{div} E=-\nabla n-\operatorname{div}(n E)-\operatorname{div}\left(E^{\mathrm{T}}-E\right)-\nabla^{-1} \mathcal{S},
$$

and

$$
\begin{aligned}
\|\operatorname{div} E\|_{L^{2}}^{2} & \leq C\left(\|\nabla n\|_{L^{2}}^{2}+\left\|\nabla\left(E^{\mathrm{T}}-E\right)\right\|_{L^{2}}^{2}+\|\nabla(n E)\|_{L^{2}}^{2}+\|E \nabla E\|_{L^{2}}^{2}\right) \\
& \leq C\left\|\nabla\left(n, E^{\mathrm{T}}-E\right)\right\|_{L^{2}}^{2}+C \delta\|\nabla E\|_{L^{2}}^{2},
\end{aligned}
$$

and

$$
\begin{aligned}
\|\nabla \operatorname{div} E\|_{L^{2}}^{2} & \leq C\left(\left\|\nabla^{2} n\right\|_{L^{2}}^{2}+\left\|\nabla^{2}\left(E^{\mathrm{T}}-E\right)\right\|_{L^{2}}^{2}+\left\|\nabla^{2}(n E)\right\|_{L^{2}}^{2}+\|\nabla(E \nabla E)\|_{L^{2}}^{2}\right) \\
& \leq C\left\|\nabla^{2}\left(n, E^{\mathrm{T}}-E\right)\right\|_{L^{2}}^{2}+C \delta\left\|\nabla^{2} E\right\|_{L^{2}}^{2} .
\end{aligned}
$$

From the above estimate, we may deduce from (2.41) that

$$
\begin{aligned}
\|\nabla E\|_{L^{2}}^{2} & \leq\|\operatorname{div} E\|_{L^{2}}^{2}+\|\operatorname{curl} E\|_{L^{2}}^{2} \\
& \leq C\left\|\nabla\left(n, E^{\mathrm{T}}-E\right)\right\|_{L^{2}}^{2}+C \delta\|\nabla E\|_{L^{2}}^{2}+\|E \nabla E\|_{L^{2}}^{2} \\
& \leq C\left\|\nabla\left(n, E^{\mathrm{T}}-E\right)\right\|_{L^{2}}^{2}+C \delta\|\nabla E\|_{L^{2}}^{2},
\end{aligned}
$$




$$
\begin{aligned}
\left\|\nabla^{2} E\right\|_{L^{2}}^{2} & \leq\left\|\nabla^{2} \operatorname{div} E\right\|_{L^{2}}^{2}+\left\|\nabla^{2} \operatorname{curl} E\right\|_{L^{2}}^{2} \\
& \leq C\left\|\nabla^{2}\left(n, E^{\mathrm{T}}-E\right)\right\|_{L^{2}}^{2}+C \delta\left\|\nabla^{2} E\right\|_{L^{2}}^{2}+\|\nabla(E \nabla E)\|_{L^{2}}^{2} \\
& \leq C\left\|\nabla^{2}\left(n, E^{\mathrm{T}}-E\right)\right\|_{L^{2}}^{2}+C \delta\left\|\nabla^{2} E\right\|_{L^{2}}^{2},
\end{aligned}
$$

and

$$
\begin{aligned}
\left\|\nabla^{3} E\right\|_{L^{2}}^{2} & \leq\|\nabla \operatorname{div} E\|_{L^{2}}^{2}+\|\nabla \operatorname{curl} E\|_{L^{2}}^{2} \\
& \leq C\left\|\nabla^{3}\left(n, E^{\mathrm{T}}-E\right)\right\|_{L^{2}}^{2}+C \delta\left\|\nabla^{3} E\right\|_{L^{2}}^{2}+\left\|\nabla^{2}(E \nabla E)\right\|_{L^{2}}^{2} \\
& \leq C\left\|\nabla^{3}\left(n, E^{\mathrm{T}}-E\right)\right\|_{L^{2}}^{2}+C \delta\left\|\nabla^{3} E\right\|_{L^{2}}^{2} .
\end{aligned}
$$

Thus, the proof of Lemma 2.5 is completed.

Now we are in a position to verify (2.4). Since $\delta \geq 0$ is sufficiently small, from Lemma (2.1)-(2.5), we can choose a constant $D_{1}>0$ suitably large such that

$$
\begin{aligned}
& \frac{\mathrm{d}}{\mathrm{d} t}\left\{D_{1}\|(n, v, E)\|_{H^{3}}^{2}+\langle\operatorname{div} v,-n\rangle+\sum_{1 \leq k \leq 2}\left\langle\nabla^{k} v, \nabla \nabla^{k} n\right\rangle-\left\langle\mathcal{W}, E^{\mathrm{T}}-E\right\rangle\right. \\
& \left.+\sum_{0 \leq k \leq 1}\left\langle\nabla^{k} \mathcal{W}, \nabla^{2} \nabla^{k}\left(E^{\mathrm{T}}-E\right)\right\rangle\right\}+C\left(\|\nabla(n, E)\|_{H^{2}}^{2}+\|v\|_{H^{4}}^{2}\right) \leq 0,
\end{aligned}
$$

for any $t \geq 0$, which implies

$$
\|(n, v, E)\|_{H^{3}}^{2}+\int_{0}^{t}\left(\|\nabla(n, E)(\tau)\|_{H^{2}}^{2}+\|v(\tau)\|_{H^{4}}^{2}\right) \mathrm{d} \tau \leq C\left\|\left(n_{0}, v_{0}, E_{0}\right)\right\|_{H^{3}}^{2},
$$

since

$$
\begin{aligned}
& \left\{D_{1}\|(n, v, E)\|_{H^{3}}^{2}+\langle\operatorname{div} v,-n\rangle+\sum_{1 \leq k \leq 2}\left\langle\nabla^{k} v, \nabla \nabla^{k} n\right\rangle-\left\langle\mathcal{W}, E^{\mathrm{T}}-E\right\rangle\right. \\
& \left.+\sum_{0 \leq k \leq 1}\left\langle\nabla^{k} \mathcal{W}, \nabla^{2} \nabla^{k}\left(E^{\mathrm{T}}-E\right)\right\rangle\right\} \sim\|(n, v, E)\|_{H^{3}}^{2} .
\end{aligned}
$$

\section{Decay Estimates}

In this section, we show the decay estimates of the solution to the problem (2.2) in low-frequency regime.

\subsection{Decay Estimates for the Linearized Problem}

We note that the linearized system (2.2) ${ }_{1}$ and (2.36) depend only on $(n, \operatorname{div} v)$ while the linearized system (2.40)-(2.43) depend only on $\left(\mathcal{W}, E^{\mathrm{T}}-E\right)$. Denote by $\Lambda^{s}$ the pseudodifferential operator is defined by

$$
\Lambda^{s} u=\mathcal{F}^{-1}\left(|\xi|^{s} \hat{u}(\xi)\right)
$$

and let

$$
\begin{gathered}
d=\Lambda^{-1} \operatorname{div} v, \\
\omega=\Lambda^{-1} \mathcal{W}=\Lambda^{-1} \operatorname{curl} v,
\end{gathered}
$$


be the "compressible part" and "incompressible part" respectively. We finally obtain

$$
\begin{gathered}
n_{t}+\Lambda d=f-v \cdot \nabla n, \\
d_{t}-(2 \mu+\lambda) \Delta d+d-2 \Lambda n=\Lambda^{-1} \operatorname{div}_{1},
\end{gathered}
$$

and

$$
\begin{gathered}
\left(E^{\mathrm{T}}-E\right)_{t}+\Lambda \omega=h^{\mathrm{T}}-h-v \cdot \nabla\left(E^{\mathrm{T}}-E\right), \\
\omega_{t}+\omega-\mu \Delta \omega-\Lambda\left(E^{\mathrm{T}}-E\right)=\Lambda^{-1} \operatorname{curl} g+\Lambda^{-1} \mathcal{S} .
\end{gathered}
$$

Indeed, as the definition of $d, \omega$ and the relation

$$
v=-\Lambda^{-1} \nabla d-\Lambda^{-1} \operatorname{div} \omega,
$$

involve pseudodifferential operators of degree zero, the estimates in space $H^{l}\left(\mathbb{R}^{3}\right)$ for the original function $v$ will be the same as for $(d, \omega)$.

Here, we just discuss the system (3.1), since the system (3.2) is the same as system (3.1). To use the estimates of the linear problem for the nonlinear system (3.1) and (3.2), we rewrite the solution of (3.1) as

$$
U(t)=K(t) U_{0}+\int_{0}^{t} K(t-\tau) G(\tau) \mathrm{d} \tau, \quad t \geq 0,
$$

where

$$
\begin{aligned}
U^{1} & =[n, d]^{\mathrm{T}}, \quad U_{0}^{1}=\left[n_{0}, d_{0}\right]^{\mathrm{T}}, \\
U^{2} & =\left[\omega, E^{\mathrm{T}}-E\right]^{\mathrm{T}}, \quad U_{0}^{2}=\left[\omega_{0},\left(E^{\mathrm{T}}-E\right)_{0}\right]^{\mathrm{T}}, \\
G^{1} & =\left[f-v \cdot \nabla n, \Lambda^{-1} \operatorname{divg}_{1}\right]^{\mathrm{T}}, \\
G^{2} & =\left[\Lambda^{-1} \operatorname{curl} g+\Lambda^{-1} \mathcal{S}, h^{\mathrm{T}}-h-v \cdot \nabla\left(E^{\mathrm{T}}-E\right)\right]^{\mathrm{T}} .
\end{aligned}
$$

And there $K(t)$ is the solution semigroup defined by $K(t)=e^{-t B}, t>0$ with $B$ being a matrix-valued differential operator given by

$$
\begin{gathered}
B_{1}=\left(\begin{array}{cc}
0 & \Lambda \\
-2 \Lambda & -(2 \mu+\lambda) \Delta+1
\end{array}\right), \\
B_{2}=\left(\begin{array}{cc}
-\mu \Delta+1 & -\Lambda \\
\Lambda & 0
\end{array}\right),
\end{gathered}
$$

and $K(t) f=\mathcal{F}^{-1}\left(e^{-t B(\xi)} \hat{f}(\xi)\right)$, we get

$$
\begin{gathered}
\hat{B}_{1}=\left(\begin{array}{cc}
0 & |\xi| \\
-2 \xi & (2 \mu+\lambda)|\xi|^{2}+1
\end{array}\right), \\
\hat{B}_{2}=\left(\begin{array}{cc}
\mu|\xi|^{2}+1 & -|\xi| \\
|\xi| & 0
\end{array}\right) .
\end{gathered}
$$

Now we need to consider the following linearized system (3.1), $i=1,2$,

$$
U_{t}^{i}=B_{i} U^{i} .
$$

Applying the Fourier transform to system (3.3), we have 


$$
\partial_{t} \hat{U}=\hat{B}(\xi) \hat{U}, \quad \hat{U}(0)=\hat{U}_{0} .
$$

By using the Fourier transform, we can get linearized system (3.1)

$$
\left\{\begin{array}{l}
\hat{n}_{t}+|\xi| \hat{d}=0, \\
\hat{d}_{t}+(2 \mu+\lambda)|\xi|^{2} \hat{d}+\hat{d}-2|\xi| \hat{n}=0,
\end{array}\right.
$$

and

$$
\left\{\begin{array}{l}
\left(\hat{E}^{\mathrm{T}}-\hat{E}\right)_{t}+|\xi| \hat{\omega}=0, \\
\hat{\omega}_{t}+\left(\mu|\xi|^{2}+1\right) \hat{\omega}+\hat{d}-2|\xi|\left(\hat{E}^{\mathrm{T}}-\hat{E}\right)=0 .
\end{array}\right.
$$

From (3.5), we have

$$
\frac{1}{2} \frac{\mathrm{d}}{\mathrm{d} t}\left\{2|\hat{n}|^{2}+|\hat{d}|^{2}\right\}+(2 \mu+\lambda)|\xi|^{2}|\hat{d}|^{2}+|\hat{d}|^{2}=0
$$

Multiplying $(3.5)_{2}$ by $\hat{n}$,

$$
\frac{\mathrm{d}}{\mathrm{d} t}(\hat{n} \hat{d})+(2 \mu+\lambda)|\xi|^{2} \hat{n} \hat{d}+\hat{n} \hat{d}-2|\xi|^{2}|\hat{n}|^{2}+|\xi||\hat{d}|^{2}=0 .
$$

Multiplying (3.8) by $-\mu|\xi|$ and (3.7) to get

$$
\begin{aligned}
& \frac{1}{2} \frac{\mathrm{d}}{\mathrm{d} t}\left\{2|\hat{n}|^{2}+|\hat{d}|^{2}-2 \mu|\xi|(\hat{n} \hat{d})\right\}+(\mu+\lambda)|\xi|^{2}|\hat{d}|^{2}+|\hat{d}|^{2}+2 \mu|\xi|^{2}|\hat{n}|^{2} \\
& =(2 \mu+\lambda)|\xi|^{3} \hat{n} \hat{d}+\mu|\xi| \hat{n} \hat{d} .
\end{aligned}
$$

Similarly, from (3.10), we get

$$
\frac{1}{2} \frac{\mathrm{d}}{\mathrm{d} t}\left\{|\hat{\omega}|^{2}+\left|\hat{E}^{\mathrm{T}}-\hat{E}\right|^{2}\right\}+\left(\mu|\xi|^{2}+1\right)|\hat{\omega}|^{2}=0
$$

and

$$
\frac{\mathrm{d}}{\mathrm{d} t}\left(\hat{\omega}\left(\hat{E}^{\mathrm{T}}-\hat{E}\right)\right)+\left(\mu|\xi|^{2}+1\right) \hat{\omega}\left(\hat{E}^{\mathrm{T}}-\hat{E}\right)-|\xi|\left|\hat{E}^{\mathrm{T}}-\hat{E}\right|^{2}+|\xi||\hat{\omega}|^{2}=0
$$

(3.10) $-\frac{\mu}{2}|\xi| \times(3.11)$ yields

$$
\begin{aligned}
& \frac{1}{2} \frac{\mathrm{d}}{\mathrm{d} t}\left\{|\hat{\omega}|^{2}+\left|\hat{E}^{\mathrm{T}}-\hat{E}\right|^{2}-\mu|\xi| \hat{\omega}\left(\hat{E}^{\mathrm{T}}-\hat{E}\right)\right\}+\frac{\mu}{2}|\xi|^{2}|\hat{\omega}|^{2}+\frac{\mu}{2}|\xi|^{2}\left|\hat{E}^{\mathrm{T}}-\hat{E}\right|^{2} \\
& =\frac{\mu^{2}}{2}|\xi|^{3}\left(\hat{E}^{\mathrm{T}}-\hat{E}\right)+\frac{\mu}{2}|\xi| \hat{\omega}\left(\hat{E}^{\mathrm{T}}-\hat{E}\right) .
\end{aligned}
$$

Let $\mathcal{L}_{1}^{2}=\mathcal{L}_{1}^{2}(\xi, t)$ and $\mathcal{L}_{2}^{2}=\mathcal{L}_{2}^{2}(\xi, t)$ be Lyapunov-type functionals defined by

$$
\mathcal{L}_{1}^{2}=2|\hat{n}|^{2}+|\hat{d}|^{2}-2 \mu|\xi|(\hat{n} \hat{d})
$$

and

$$
\mathcal{L}_{2}^{2}=|\hat{\omega}|^{2}+\left|\hat{E}^{\mathrm{T}}-\hat{E}\right|^{2}-\mu|\xi| \hat{\omega}\left(\hat{E}^{\mathrm{T}}-\hat{E}\right)
$$

Then it is clear that there exists a small enough constant $c>0$, such that for any $|\xi| \leq c$, 


$$
\frac{1}{c} \mathcal{L}_{1}^{2} \leq 2|\hat{n}|^{2}+|\hat{d}|^{2} \leq c
$$

and

$$
\frac{1}{c} \mathcal{L}_{2}^{2} \leq 2|\hat{\omega}|^{2}+\left|\hat{E}^{\mathrm{T}}-\hat{E}\right|^{2} \leq c .
$$

From (3.9) and (3.16), we have

$$
\frac{1}{2} \frac{\mathrm{d}}{\mathrm{d} t} \mathcal{L}_{1}^{2}+(\mu+\lambda)|\xi|^{2}|\hat{d}|^{2}+|\hat{d}|^{2}+2 \mu|\xi|^{2}|\hat{n}|^{2}=(2 \mu+\lambda)|\xi|^{3} \hat{n} \hat{d}+\mu|\xi| \hat{n} \hat{d}
$$

and

$$
\frac{1}{2} \frac{\mathrm{d}}{\mathrm{d} t} \mathcal{L}_{2}^{2}+\frac{\mu}{2}|\xi|^{2}|\hat{\omega}|^{2}+\frac{\mu}{2}|\xi|^{2}\left|\hat{E}^{\mathrm{T}}-\hat{E}\right|^{2}=\frac{\mu^{2}}{2}|\xi|^{3}\left(\hat{E}^{\mathrm{T}}-\hat{E}\right)+\frac{\mu}{2}|\xi| \hat{\omega}\left(\hat{E}^{\mathrm{T}}-\hat{E}\right) .
$$

Using the Young inequality, we deduce that there exists a positive $C$ and a sufficient small constant $C_{0}>0$ such that for any $|\xi| \leq C_{0}$,

$$
\frac{\mathrm{d}}{\mathrm{d} t} \mathcal{L}_{i}^{2}+|\xi|^{2} \mathcal{L}_{i}^{2} \leq 0, \quad \text { for } i=1,2
$$

Therefore

$$
\mathcal{L}_{i}^{2}(\xi, t) \leq e^{-c|\xi|^{2} t} \mathcal{L}_{i}^{2}(\xi, 0)
$$

where $\mathcal{L}_{1}^{2}(\xi, 0) \sim\left|\left(\hat{n}_{0}, \hat{d}_{0}\right)\right|^{2}$ and $\mathcal{L}_{2}^{2}(\xi, 0) \sim\left|\left(\hat{\omega}_{0}, \hat{E}_{0}^{\mathrm{T}}-\hat{E}_{0}\right)\right|^{2}$.

We define a low frequency and high frequency decomposition $\left(f_{l}(x), f_{h}(x)\right)$ for a function $f(x) \in L^{2}\left(\mathbb{R}^{3}\right)$

$$
f_{l}(x)=\mathcal{F}^{-1}\left(\varphi_{0}(\xi) \hat{f}(\xi)\right), \quad f_{h}(x)=\mathcal{F}^{-1}\left(\varphi_{0}(\xi) \hat{f}(\xi)\right) .
$$

Let $\varphi_{0}(\xi)$ be a function in $C_{0}^{\infty}\left(\mathbb{R}^{3}\right)$ and some chosen constant $R$ such that,

$$
\varphi_{0}(\xi)= \begin{cases}1, & |\xi|<\frac{R}{2}, \\ 0, & |\xi|>R .\end{cases}
$$

Thus, we get

$$
f(x)=f_{l}(x)+f_{h}(x) .
$$

Then

$$
U_{l}(t)=K(t)_{l} U_{0}+\int_{0}^{t} K_{l}(t-\tau) G(\tau) \mathrm{d} \tau, \quad t \geq 0 .
$$

We show the decay estimates of $U_{l}(t)$ as follows.

Lemma 3.1 Let $\left(n_{0}, d_{0}\right) \in H^{3}\left(\mathbb{R}^{3}\right)$, then for $0 \leq k \leq 3$, the following decay

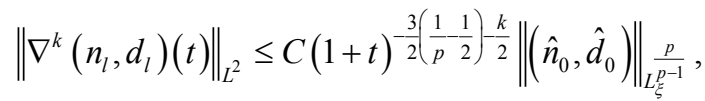

$$
\begin{aligned}
& \left\|\nabla^{k}\left(\omega_{l}, E_{l}^{\mathrm{T}}-E_{l}\right)(t)\right\|_{L^{2}} \leq C(1+t)^{-\frac{3}{2}\left(\frac{1}{p}-\frac{1}{2}\right)-\frac{k}{2}}\left\|\left(\hat{\omega}_{0}, \hat{E}_{0}^{\mathrm{T}}-\hat{E}_{0}\right)\right\|_{L_{\xi}^{p-1}}, \\
& \left\|\nabla^{k}\left(n_{l}, d_{l}\right)(t)\right\|_{L^{\infty}} \leq C(1+t)^{-\frac{3}{2 p}-\frac{k}{2}}\left\|\left(\hat{n}_{0}, \hat{d}_{0}\right)\right\|_{L_{E}^{p-1}},
\end{aligned}
$$




$$
\left\|\nabla^{k}\left(\omega_{l}, E_{l}^{\mathrm{T}}-E_{l}\right)(t)\right\|_{L^{\infty}} \leq C(1+t)^{-\frac{3}{2 p}-\frac{k}{2}}\left\|\left(\hat{\omega}_{0}, \hat{E}_{0}^{\mathrm{T}}-\hat{E}_{0}\right)\right\|_{L_{\xi}^{p-1}} .
$$

Proof. By using the Plancherel theorem and the Hölder inequality, we get

$$
\begin{aligned}
\left\|\nabla^{k}\left(n_{l}, d_{l}\right)(t)\right\|_{L^{2}} & =\left\|\nabla^{k}\left(\hat{n}_{l}, \hat{d}_{l}\right)(t)\right\|_{L^{2}} \\
& \leq C\left(\int_{|\xi| \leq C_{0}}|\xi|^{2 k} \mathcal{L}_{1}^{2}(\xi, t) \mathrm{d} \xi\right)^{\frac{1}{2}} \\
& \leq C\left(\int_{|\xi| \leq C_{0}}|\xi|^{2 k} e^{-c|\xi|^{2} t} \mathcal{L}_{1}^{2}(\xi, 0) \mathrm{d} \xi\right)^{\frac{1}{2}} \\
& \leq C\left(\int_{|\xi| \leq C_{0}}|\xi|^{\frac{2 k p}{2-p}} e^{-\frac{c p}{2-p}|\xi|^{2} t} \mathrm{~d} \xi\right)^{\frac{2-p}{2 p}}\left(\int_{|\xi| \leq C_{0}}\left(\mathcal{L}_{1}^{2}(\xi, 0)\right)^{\frac{p}{2(p-1)}} \mathrm{d} \xi\right)^{\frac{p-1}{p}} \\
& \leq C(1+t)^{-\frac{3}{2}\left(\frac{1}{p}-\frac{1}{2}\right)-\frac{k}{2}}\left\|\left(\hat{n}_{0}, \hat{d}_{0}\right)\right\|_{L_{\xi}^{p-1}} .
\end{aligned}
$$

Similarly, we can get (3.24)-(3.27).

Next, we consider decay rates for the nonlinear system.

\subsection{Decay Estimates for the Nonlinear Problem}

Lemma 3.2 It holds that for any integer $k \geq 0$,

$$
\begin{aligned}
& \left\|\nabla^{k}\left(n_{l}, d_{l}, \omega_{l}, E_{l}^{\mathrm{T}}-E_{l}\right)(t)\right\|_{L^{2}} \\
& \leq C(1+t)^{-\frac{3}{2}\left(\frac{1}{p}-\frac{1}{2}\right)-\frac{k}{2}}\left\|\left(n_{0}, d_{0}, \omega_{0},\left(E^{\mathrm{T}}-E\right)\right)\right\|_{L^{p}} \\
& \quad+C \delta \int_{0}^{\frac{t}{2}}(1+t-\tau)^{-\frac{3}{4}-\frac{k}{2}}\left(\|n(\tau)\|_{L^{2}}+\left\|\nabla\left(n, v, E^{\mathrm{T}}-E\right)(\tau)\right\|_{H^{1}}\right) \mathrm{d} \tau \\
& \quad+C \int_{\frac{t}{2}}^{t}(1+t-\tau)^{-\frac{1}{4}-\frac{k}{2}}\left(\|n(\tau)\|_{L^{2}}^{2}+\left\|\nabla\left(n, v, E^{\mathrm{T}}-E\right)(\tau)\right\|_{H^{1}}^{2}\right) \mathrm{d} \tau,
\end{aligned}
$$

and

$$
\begin{aligned}
& \left\|\nabla^{k}\left(n_{l}, d_{l}, \omega_{l}, E_{l}^{\mathrm{T}}-E_{l}\right)(t)\right\|_{L^{\infty}} \\
& \leq C(1+t)^{-\frac{3}{2 p}-\frac{k}{2}}\left\|\left(n_{0}, d_{0}, \omega_{0},\left(E^{T}-E\right)\right)\right\|_{L^{p}} \\
& \quad+C \delta \int_{0}^{\frac{t}{2}}(1+t-\tau)^{-\frac{3}{2}-\frac{k}{2}}\left(\|n(\tau)\|_{L^{2}}+\left\|\nabla\left(n, v, E^{\mathrm{T}}-E\right)(\tau)\right\|_{H^{1}}\right) \mathrm{d} \tau \\
& \quad+C \int_{\frac{t}{2}}^{t}(1+t-\tau)^{-\frac{3}{4}-\frac{k}{2}}\left(\|n(\tau)\|_{L^{2}}^{2}+\left\|\nabla\left(n, v, E^{\mathrm{T}}-E\right)(\tau)\right\|_{H^{1}}^{2}\right) \mathrm{d} \tau .
\end{aligned}
$$

Proof. By using the Duhamel principle

$$
U_{l}^{i}(t)=K_{l}^{i}(t) U^{i}(0)+\int_{0}^{t} K_{l}^{i}(t-\tau) G^{i}(\tau) \mathrm{d} \tau, \quad i=1,2 .
$$

By using (3.3) and the Hausdorff-Young inequality, the nonlinear source terms can be estimates as follows:

$$
\left\|\left(G^{1}, G^{2}\right)\right\|_{L^{\infty}} \leq C \delta\left(\|n\|_{L^{2}}+\left\|\nabla\left(n, v, E^{\mathrm{T}}-E\right)\right\|_{H^{1}}\right),
$$




$$
\begin{gathered}
\left\|\left(G^{1}, G^{2}\right)\right\|_{L^{3}} \leq C\left(\|n\|_{L^{2}}^{2}+\left\|\nabla\left(n, v, E^{\mathrm{T}}-E\right)\right\|_{H^{1}}^{2}\right), \\
\left\|\left(G^{1}, G^{2}\right)\right\|_{L^{2}} \leq C\left(\|n\|_{L^{2}}^{2}+\left\|\nabla\left(n, v, E^{\mathrm{T}}-E\right)\right\|_{H^{1}}^{2}\right) .
\end{gathered}
$$

Put these estimates into (3.30), this completes the proof of Lemma 3.2.

\subsection{Optimal Decay Estimates}

Now we will show the proof of Proposition 2.2. Define the temporal energy functional

$$
\mathcal{E}(t)=D_{2}\|\nabla(n, v, E)\|_{H^{2}}^{2}+\sum_{1 \leq k \leq 2}\left\langle\nabla^{k} v, \nabla \nabla^{k} n\right\rangle+\sum_{0 \leq k \leq 1}\left\langle\nabla^{k} \mathcal{W}, \nabla^{2} \nabla^{k}\left(E^{\mathrm{T}}-E\right)\right\rangle,
$$

for any $0 \leq t \leq T$, where it should be mentioned that $\mathcal{E}(t)$ is equivalent to $\|\nabla(n, v, E)\|_{H^{2}}^{2}$, we get

$$
\frac{\mathrm{d}}{\mathrm{d} t} \mathcal{E}(t)+C\left(\left\|\nabla^{2}(n, E)\right\|_{H^{1}}^{2}+\|\nabla v\|_{H^{3}}^{2}\right) \leq C \delta\|\nabla(n, E)\|_{L^{2}}^{2} .
$$

From (3.23), we have

$$
\|\nabla(n, v, E)\|_{L^{2}}^{2} \leq\left\|\nabla\left(n_{l}, v_{l}, E_{l}\right)\right\|_{L^{2}}^{2}+\left\|\nabla\left(n_{h}, v_{h}, E_{h}\right)\right\|_{L^{2}}^{2} .
$$

Adding $\left\|\nabla\left(n_{l}, v_{l}, E_{l}\right)\right\|_{L^{2}}^{2}$ to both of the inequality and using the smallness of $\delta$, we have

$$
\frac{\mathrm{d}}{\mathrm{d} t} \mathcal{E}(t)+D_{3} \mathcal{E}(t) \leq C\left\|\nabla\left(n_{l}, v_{l}, E_{l}\right)\right\|_{L^{2}}^{2} .
$$

Here $D_{3}$ can be large enough. If we define

$$
\begin{aligned}
\mathcal{H}(t)= & \sup _{0 \leq \tau \leq t}\left\{(1+\tau)^{\frac{5}{4}}\left(\|n\|_{L^{2}}+\left\|\nabla\left(n, v, E^{\mathrm{T}}-E\right)\right\|_{L^{2}}\right)\right. \\
& \left.+(1+\tau)^{\frac{7}{4}}\left(\|n\|_{L^{2}}+\left\|\nabla^{2}\left(n, v, E^{\mathrm{T}}-E\right)\right\|_{L^{2}}\right)\right\} .
\end{aligned}
$$

Then, by using (3.38) and Lemma 3.2 that for any $0 \leq k \leq 3$,

$$
\begin{aligned}
& \left\|\nabla^{k}\left(n_{l}, d_{l}, E_{l}^{\mathrm{T}}-E_{l}\right)(t)\right\|_{L^{2}} \\
& \leq C(1+t)^{-\frac{3}{4}-\frac{k}{2}}\left\|\left(n_{0}, d_{0},\left(E_{0}^{\mathrm{T}}-E_{0}\right)\right)\right\|_{L^{1}} \\
& +C \delta \int_{0}^{\frac{t}{2}}(1+t-\tau)^{-\frac{3}{4}-\frac{k}{2}}\left(\|n(\tau)\|_{L^{2}}+\left\|\nabla\left(n, v, E^{\mathrm{T}}-E\right)(\tau)\right\|_{H^{1}}\right) \mathrm{d} \tau \\
& +C \int_{\frac{t}{2}}^{t}(1+t-\tau)^{-\frac{1}{4}-\frac{k}{2}}\left(\|n(\tau)\|_{L^{2}}^{2}+\left\|\nabla\left(n, v, E^{\mathrm{T}}-E\right)(\tau)\right\|_{H^{1}}^{2}\right) \mathrm{d} \tau \\
& \leq C(1+t)^{-\frac{3}{4}-\frac{k}{2}}\left\|\left(n_{0}, d_{0},\left(E_{0}^{\mathrm{T}}-E_{0}\right)\right)\right\|_{L^{1}}+C \delta \mathcal{H}(t) \int_{0}^{\frac{t}{2}}(1+t-\tau)^{-\frac{3}{4}-\frac{k}{2}}(1+\tau)^{-\frac{5}{4}} \mathrm{~d} \tau \\
& \quad+C\left\|\left(n_{0}, d_{0},\left(E_{0}^{\mathrm{T}}-E_{0}\right)\right)\right\|_{H^{3}} \mathcal{H}(t) \int_{\frac{t}{2}}^{t}(1+t-\tau)^{-\frac{1}{4}-\frac{k}{2}}(1+\tau)^{-\frac{5}{4}-\frac{1}{2}} \mathrm{~d} \tau \\
& \leq C(1+t)^{-\frac{3}{4}-\frac{k}{2}}\left\{\left\|\left(n_{0}, d_{0},\left(E_{0}^{\mathrm{T}}-E_{0}\right)\right)\right\|_{L^{1}}+\left(\delta+\delta_{0}\right) \mathcal{H}(t)\right\} .
\end{aligned}
$$

From (3.37) and (3.39), we get 
$\mathcal{E}(t)$

$$
\begin{aligned}
& \leq e^{-c t} \mathcal{E}(0)+\left\{\left\|\left(n_{0}, d_{0},\left(E_{0}^{\mathrm{T}}-E_{0}\right)\right)\right\|_{L^{1}}^{2}+\left(\delta^{2}+\delta_{0}^{2}\right) \mathcal{H}(t)^{2}\right\} \int_{0}^{t} e^{-c(t-\tau)}(1+\tau)^{-\frac{5}{2}} \mathrm{~d} \tau(3.40) \\
& \leq C(1+t)^{-\frac{5}{2}}\left(\mathcal{E}(0)+\left\|\left(n_{0}, d_{0},\left(E_{0}^{\mathrm{T}}-E_{0}\right)\right)\right\|_{L^{1}}^{2}+\left(\delta^{2}+\delta_{0}^{2}\right) \mathcal{H}(t)^{2}\right)
\end{aligned}
$$

and also can get

$$
\begin{aligned}
& \left\|\nabla\left(n, d, E^{\mathrm{T}}-E\right)(t)\right\|_{H^{2}} \\
& \leq C(1+t)^{-\frac{5}{4}}\left(\left\|\left(n_{0}, d_{0},\left(E_{0}^{\mathrm{T}}-E_{0}\right)\right)\right\|_{L^{1} \cap H^{3}}+\left(\delta+\delta_{0}\right) \mathcal{H}(t)\right) .
\end{aligned}
$$

Therefore we obtain that for any $0 \leq k \leq 3$,

$$
\begin{aligned}
& \left\|\nabla^{k}\left(n, d, E^{\mathrm{T}}-E\right)(t)\right\|_{L^{2}} \\
& \leq C(1+t)^{-\frac{3}{4}-\frac{k}{2}}\left(\left\|\left(n_{0}, d_{0},\left(E_{0}^{\mathrm{T}}-E_{0}\right)\right)\right\|_{L^{1} \cap H^{3}}+\left(\delta+\delta_{0}\right) \mathcal{H}(t)\right) .
\end{aligned}
$$

Noticing $\mathcal{H}(t)$, with the smallness of $\delta$, we have

$$
\mathcal{H}(t) \leq C\left\|\left(n_{0}, d_{0},\left(E_{0}^{\mathrm{T}}-E_{0}\right)\right)\right\|_{L^{\mathrm{I}} \cap H^{3}} .
$$

Considering (2.35) and Lemma 2.5, we get

$$
\begin{aligned}
\|E\|_{L^{2}} & \leq C\left\|\Lambda^{-1} \operatorname{div} E\right\|_{L^{2}}+\left\|\Lambda^{-1} \operatorname{curl} E\right\|_{L^{2}} \\
& \leq C\left\|\left(n, E^{\mathrm{T}}-E\right)\right\|_{L^{2}}+C\left\|n E^{\mathrm{T}}\right\|_{L^{2}}+C\left\|\Lambda^{-1}(E \nabla E)\right\|_{L^{2}} \\
& \leq C(1+t)^{-\frac{3}{4}}\left\|\left(n_{0}, d_{0},\left(E_{0}^{\mathrm{T}}-E_{0}\right)\right)\right\|_{L^{1} \cap H^{3}}+C\|(E \nabla E)\|_{L^{\frac{6}{5}}} \\
& \leq C(1+t)^{-\frac{3}{4}}\left\|\left(n_{0}, d_{0},\left(E_{0}^{\mathrm{T}}-E_{0}\right)\right)\right\|_{L^{1} \cap H^{3}} .
\end{aligned}
$$

Similarly, by Lemma 3.2 we can obtain

$$
\left\|\left(n, d, E^{\mathrm{T}}-E\right)(t)\right\|_{L^{\infty}} \leq C(1+t)^{-\frac{3}{2}}\left\|\left(n_{0}, d_{0},\left(E_{0}^{\mathrm{T}}-E_{0}\right)\right)\right\|_{L^{1} \cap H^{3}} .
$$

From (2.2) and use the estimates above, we get

$$
\begin{aligned}
\left\|\partial_{t}(n, v, E)\right\|_{L^{2}} \leq & C\left\{\|\operatorname{div} v\|_{L^{2}}+\|f-v \cdot \nabla n\|_{L^{2}}+\|\Delta v\|_{L^{2}}+\|\nabla \operatorname{div} v\|_{L^{2}}\right. \\
& \left.+\|\nabla n\|_{L^{2}}+\|\nabla E\|_{L^{2}}+\|g\|_{L^{2}}+\|\nabla v\|_{L^{2}}+\|h-v \cdot \nabla E\|_{L^{2}}\right\} \\
\leq & C(1+t)^{-\frac{3}{4}}
\end{aligned}
$$

\section{Conflicts of Interest}

The author declares no conflicts of interest regarding the publication of this paper.

\section{References}

[1] Hu, X. and Wu, G. (2013) Global Existence and Optimal Decay Rates for Three-Dimensional Compressible Viscoelastic Flows. SIAM Journal on Mathematical Analysis, 45, 2815-2833. https://doi.org/10.1137/120892350 
[2] Hu, X.P. and Masmoudi, N. (2017) Global Solutions to Repulsive Hookean Elastodynamics. Archive for Rational Mechanics and Analysis, 223, 543-590. https://doi.org/10.1007/s00205-016-1039-2

[3] Hu, X. and Wang, D. (2011) Global Existence for the Multi-Dimensional Compressible Viscoelastic Flows. Journal of Differential Equations, 250, 1200-1231. https://doi.org/10.1016/j.jde.2010.10.017

[4] Qian, J. and Zhang, Z. (2010) Global Well-Posedness for the Compressible Viscoelastic Fluids near Equilibrium. Archive for Rational Mechanics and Analysis, 198, 835-868. https://doi.org/10.1007/s00205-010-0351-5

[5] Hu, X. and Wang, D. (2015) The Initial-Boundary Value Problem for the Compressible Viscoelastic Flows. Discrete and Continuous Dynamical Systems, 35, 917-934. https://doi.org/10.3934/dcds.2015.35.917

[6] Huang, X.D. (2017) Existence and Uniqueness of Weak Solutions of the Compressible Spherically Symmetric Navier-Stokes Equations. Journal of Differential Equations, 262, 1341-1358. https://doi.org/10.1016/j.jde.2016.10.013

[7] Qian, J. (2011) Initial Boundary Value Problems for the Compressible Viscoelastic Fluid. Journal of Differential Equations, 250, 848-865.

https://doi.org/10.1016/j.jde.2010.07.026

[8] Hu, X. and Wang, D. (2010) Local Strong Solution to the Compressible Viscoelastic Flow with Large Data. Journal of Differential Equations, 249, 1179-1198. https://doi.org/10.1016/j.jde.2010.03.027

[9] Huang, X.D. and Li, J. (2016) Existence and Blow up Behavior of Global Strong Solutions to the Two-Dimensional Barotrpic Compressible Navier-Stokes System with Vacuum and Large Initial Data. Journal de Mathématiques Pures et Appliquées, 106, 123-154. https://doi.org/10.1016/j.matpur.2016.02.003

[10] Jiu, Q.S., Li, M.J. and Ye, Y.L. (2014) Global Classical Solution of the Cauchy Problem to $1 \mathrm{D}$ Compressible Navier-Stokes Equations with Large Initial Data. Journal of Differential Equations, 257, 311-350. https://doi.org/10.1016/j.jde.2014.03.014

[11] Wang, W., Wang, J. and Zhang, W. (2018) Decay Rates of the Compressible Viscoelastic Flows with Electric Potential. Journal of Mathematical Analysis and Applications, 463, 50-78. https://doi.org/10.1016/j.jmaa.2018.03.006

[12] Chemin, J.Y. and Masmoudi, N. (2001) About Lifespan of Regular Solutions of Equations Related to Viscoelastic Fluids. SIAM Journal on Mathematical Analysis, 33, 84-112. https://doi.org/10.1137/S0036141099359317

[13] Chen, Y. and Zhang, P. (2006) The Global Existence of Small Solutions to the Incompressible Viscoelastic Fluid System in 2 and 3 Space Dimensions. Communications in Partial Differential Equations, 31, 1793-1810. https://doi.org/10.1080/03605300600858960

[14] $\mathrm{Hu}, \mathrm{X}$. and $\mathrm{Wu}, \mathrm{H}$. (2015) Long-Time Behavior and Weak-Strong Uniqueness for Incompressible Viscoelastic Flows. Discrete \& Continuous Dynamical Systems, 35, 3437-3461. https://doi.org/10.3934/dcds.2015.35.3437

[15] Lei, Z., Liu, C. and Zhou, Y. (2007) Global Existence for a 2D Incopressible Viscoelastic Model with Small Strain. Communications in Mathematical Sciences, 5, 595-616. https://doi.org/10.4310/CMS.2007.v5.n3.a5

[16] Lin, F., Liu, C. and Zhang, P. (2005) On Hydrodynamics of Viscoelastic Fluids. Communications on Pure and Applied Mathematics, 58, 1437-1471. https://doi.org/10.1002/cpa.20074

[17] Lin, F. and Zhang, P. (2008) On the Initial-Boundary Value Problem of the Incom- 
pressible Viscoelastic Fluid System. Communications on Pure and Applied Mathematics, 61, 539-558. https://doi.org/10.1002/cpa.20219

[18] Zhong, X. (2017) Global Well-Posedness to the Incompressible Navier-Stokes Equations with Damping. Electronic Journal of Qualitative Theory of Differential Equations, 62, 1-9.

[19] Liu, C. and Walkington, N.J. (2001) An Eulerian Description of Fluids Containing Visco-Elastic Particles. Archive for Rational Mechanics and Analysis, 159, 229-252.

[20] Cai, X.J. and Jiu, Q.S. (2008) Weak and Strong Solutions for the Incompressible Navier-Stokes Equations with Damping. Journal of Mathematical Analysis and Applications, 343, 799-809. https://doi.org/10.1016/j.jmaa.2008.01.041

[21] Cai, X.J. and Lei, L.H. (2010) L Decay of the Incompressible Navier-Stokes Equations with Damping. Acta Mathematica Scientia, 30, 1235-1248. https://doi.org/10.1016/S0252-9602(10)60120-8

[22] Jiang, Z.H. and Zhu, M.X. (2012) The Large Time Behavior of Solutions to 3D Navier-Stokes Equations with Nonlinear Damping. Mathematical Methods in the Applied Sciences, 35, 97-102. https://doi.org/10.1002/mma.1540

[23] Qiu, H.L., Zhang, Y.C., Mei, L.Q. and Xue, C.F. (2017) A Penalty-FEM for Navier-Stokes Type Variational Inequality with Nonlinear Damping Term. Computers and Mathematics with Applications, 33.

[24] Qiu, H.L., Zhang, Y.C. and Mei, L.Q. (2017) A Mixed-FEM for Navier-Stokes Type Variational Inequality with Nonlinear Damping Term. Computers and Mathematics with Applications, 73, 2191-2207. https://doi.org/10.1016/j.camwa.2017.02.046

[25] Zhang, Y.H and Tan, Z. (2007) On the Existence of Solutions to the Navier-Stokes-Poission Equations of a Two-Dimensional Compressible Flow. Mathematical Methods in the Applied Sciences, 30, 305-329. https://doi.org/10.1002/mma.786

[26] Majda, A.J. and Bertozzi, A.L. (2002) Vorticity and Incompressible Flow. Cambridge University Press, Cambridge. 


\section{Appendix}

In this appendix, we state some useful inequalities in the Sobolev space.

Lemma 4.1 Let $f \in H^{2}\left(\mathbb{R}^{3}\right)$. Then

$$
\begin{gathered}
\|f\|_{L^{\infty}} \leq C\|\nabla f\|_{L^{2}}^{\frac{1}{2}}\|\nabla f\|_{H^{1}}^{\frac{1}{2}} \leq C\|\nabla f\|_{H^{1}}^{\frac{1}{2}}, \\
\|f\|_{L^{6}} \leq C\|\nabla f\|_{L^{2}}, \\
\|f\|_{L^{q}} \leq C\|f\|_{H^{1}}, 2 \leq q \leq 6 .
\end{gathered}
$$

Lemma 4.2 Let $m \geq 1$ be an integer, then we have

$$
\left\|\nabla^{m}(f g)\right\|_{L^{p}} \leq C\|f\|_{L^{p_{1}}}\left\|\nabla^{m} g\right\|_{L^{p^{2}}}+C\left\|\nabla^{m} f\right\|_{L^{p_{3}}}\|g\|_{L^{p^{4}}},
$$

and

$$
\left\|\nabla^{m}(f g)-f \nabla^{m} g\right\|_{L^{p}} \leq C\|\nabla f\|_{L^{p_{1}}}\left\|\nabla^{m-1} g\right\|_{L^{p_{2}}}+C\left\|\nabla^{m} f\right\|_{L^{p_{3}}}\|g\|_{L^{p_{4}}},
$$

where $p, p_{1}, p_{2}, p_{3}, p_{4} \in[1, \infty]$ and

$$
\frac{1}{p}=\frac{1}{p_{1}}+\frac{1}{p_{2}}+\frac{1}{p_{3}}+\frac{1}{p_{4}} .
$$

Proof. Please refer for instance to [26].

Lemma 4.3 Let $0<s<3,1<p<q<\infty, \frac{1}{q}+\frac{s}{3}=\frac{1}{p}$, then

$$
\left\|\Lambda^{-s} f\right\|_{L^{q}} \leq C\|f\|_{L^{p}} .
$$

Lemma 4.4 If $r_{1}>1$ and $r_{2} \in\left[0, r_{1}\right]$, then it holds that

$$
\int_{0}^{t}(1+t-\tau)^{-r_{1}}(1+t)^{-r_{2}} \mathrm{~d} \tau \leq C\left(r_{1}, r_{2}\right)(1+t)^{-r_{2}} .
$$

